\title{
Separable Codes for the Symmetric Multiple-Access Channel
}

\author{
Arkadii G. D’yachkov, Nikita A. Polyanskii, Ilya V. Vorobyev, and Vladislav Yu. Shchukin
}

\begin{abstract}
A binary matrix is called an s-separable code for the disjunctive multiple-access channel (disj-MAC) if Boolean sums of sets of $s$ columns are all distinct. The well-known issue of the combinatorial coding theory is to obtain upper and lower bounds on the rate of $s$-separable codes for the disj-MAC. In our paper, we generalize the problem and discuss upper and lower bounds on the rate of $q$-ary $s$-separable codes for models of noiseless symmetric MAC, i.e., at each time instant the output signal of MAC is a symmetric function of its $s$ input signals.
\end{abstract}

\section{Index Terms}

Multiple-access channel (MAC), separable codes, random coding method, list-decoding.

\section{INTRODUCTION}

$\mathbf{W}$ E study some combinatorial coding problems for the multiple access channel (MAC) that were motivated by two specific noiseless MAC models, corresponding to the transmission of $q$-ary symbols based on the frequency modulation method. Both models were suggested in the paper [1] and were called the $s$-user $q$-frequency MAC with (the $B$-MAC) and without (the $A-\mathrm{MAC}$ ) intensity information. Using a well-known terminology [2] of the combinatorial coding theory, we describe the $A-\mathrm{MAC}$ and the $B-\mathrm{MAC}$ coding problems along with the previously obtained results as follows.

Given arbitrary integers $2 \leq s<t / 2, q \geq 2$ and $N \geq 2$, introduce a code $X$ consisting of $t$ codewords of length $N$ over a $q$-ary alphabet. The code $X$ is called

- $s$-separable [3] code for the $A$-MAC if for any two distinct $s$-tuples of the codewords there exists a coordinate $i$, $1 \leq i \leq N$, in which the union of s elements of the first s-tuple differs from the union of s elements of the second s-tuple.

- s-separable [4] code for the $B$-MAC if for any two distinct $s$-tuples of the codewords there exists a coordinate $i$, $1 \leq i \leq N$, in which the type (or the composition) of the first s-tuple differs from the type of the second s-tuple.

- $(\leq s)$-separable [3] code for the $A$-MAC if for any $k$-tuple and any $m$-tuple, where $1 \leq k, m \leq s$, of the codewords there exists a coordinate $i, 1 \leq i \leq N$, in which the union of $k$ elements of the $k$-tuple differs from the union of $m$ elements of the m-tuple.

- $s$-frameproof code [5] if for any $s$-tuple of the codewords and every other codeword, there exists a coordinate $i, 1 \leq i \leq N$, in which the symbol of the other codeword doesn't belong to the union of s elements of the s-tuple.

- s-hash code [6], [7] if $q \geq s$ and for every $s$-tuple of the codewords there exists a coordinate $i, 1 \leq i \leq N$, in which they all are differ.

If $t^{(A)}(s, q, N)$ denote the largest size of $s$-separable codes for the $A$-MAC, then the number

$$
R^{(A)}(s, q)=\varlimsup_{N \rightarrow \infty} \frac{\ln t^{(A)}(s, q, N)}{N},
$$

is said to be the rate of $s$-separable codes for the $A$-MAC. By the similar way we define the rate $R^{(B)}(s, q)$ of $s$-separable codes for the $B$-MAC, the rate $R^{(h a s h)}(s, q)$ of $s$-hash codes, the rate $R^{(A)}(\leq s, q)$ of $(\leq s)$-separable codes and the rate $R^{(f p)}(s, q)$ of $s$-frameproof codes.

\section{A. Related Work}

Multimedia fingerprinting is a technique to trace the sources of pirate copies of copyrighted multimedia contents. Separable codes for the $A-\mathrm{MAC}$ were introduced in [3] as an efficient tool to construct codes for multimedia fingerprinting in the context of "averaging attack". Due to its importance, constructions, applications and bounds on the rate of separable codes were further investigated and discussed in many papers [8]-[10].

A. G. D'yachkov is with the Lomonosov Moscow State University, Moscow 119991, Russia (e-mail: agd-msu@yandex.ru).

N. A. Polyanskii is with the Israel Institute of Technology, Haifa 32000, Israel, and with the Skolkovo Institute of Science and Technology, Moscow 121205, Russia (e-mail: nikitapolyansky@gmail.com).

V. Yu. Shchukin is with the Institute for Information Transmission Problems, Moscow 127051, Russia (e-mail: vpike@mail.ru).

I. V. Vorobyev is with the Skolkovo Institute of Science and Technology, Moscow 121205, Russia, and also with the Moscow Institute of Physics and Technology, Dolgoprudny 141701, Russia (e-mail: vorobyev.i.v@yandex.ru).

A. G. D'yachkov, N. A. Polyanskii, V. Yu. Shchukin, and I. V. Vorobyev, are supported by the Russian Foundation for Basic Research under grant No. 16-01-00440 a. N. A. Polyanskii is supported in part by the Israel Science Foundation grant nos. 1162/15, 326/17. 
Other security models and applications related to separable codes have been considered, and various classes of codes were defined in the literature. We only mention the most significant one and refer the reader to [5], where the problem of preventing an adversary from framing an innocent user was addressed, and the definition of frameproof codes was given. The latter were studied extensively in [3], [11] $-[14]$.

Finally, hash codes have undergone study due to their applications in information retrieval, cryptography and algorithms. Different problems on hash codes were considered and developed in [6], [7], [15], [16].

Recall the well-known results emphasizing the connection between separable codes, hash codes and frameproof codes

$$
\begin{aligned}
R^{(A)}(\leq s, q) & \leq \min \left\{R^{(f p)}(s-1, q), R^{(A)}(s, q)\right\}, \\
R^{(f p)}(s, q) & \leq R^{(A)}(\leq s, q), \\
R^{(h a s h)}(s, q) & \leq R^{(f p)}(s-1, q), \quad q \geq s \geq 2,
\end{aligned}
$$

and asymptotic $(q \rightarrow \infty)$ lower and upper bounds

$$
\begin{aligned}
R^{(\text {hash })}(s, q) & \geq \frac{\ln q}{s-1}(1+o(1)), \\
R^{(f p)}(s, q) & \leq \frac{\ln q}{s}(1+o(1)) .
\end{aligned}
$$

The first and the second inequalities in (1) are simple reformulations of the corresponding evident properties of binary superimposed codes [17], [18]. The third inequality in (1) is trivially implied from the definitions. The upper bound for frameproof codes in (2) is given in [13] and is based on the same idea as an upper bound for hash codes [16], [19]. The asymptotic lower bound in (2) is an obvious corollary of the random coding lower bound proved in [6], [20]. From (1] and (2), it follows the asymptotic $(q \rightarrow \infty)$ equalities:

$$
R^{(h a s h)}(s, q) \sim \frac{\ln q}{s-1}, \quad R^{(f p)}(s, q) \sim \frac{\ln q}{s} .
$$

Moreover, recent papers [9], [10] contains proofs of the asymptotic $(q \rightarrow \infty)$ equalities:

$$
R^{(A)}(\leq 2, q) \sim \frac{2 \ln q}{3} ; \quad R^{(A)}(\leq s, q) \sim \frac{\ln q}{s-1}, \quad s \geq 3
$$

Unlike 3 and (4), the asymptotic behavior of the rates $R^{(A)}(s, q)$ and $R^{(B)}(s, q)$ of $s$-separable codes for the $A$-MAC and the $B$-MAC is unknown at present. The aim of our paper is a further development and generalizations of the given open problems.

\section{B. Outline}

The remainder of the paper is organized as follows. After introducing notations, in Section II we give formal definitions of MAC and a separable code for MAC, and describe five models of MACs, which are important for applications. In Section III we discuss the entropy upper bound on the rate of separable codes for any symmetric MAC and its known and new improvements. In particular, a combinatorial upper bound on $R^{(B)}(s, q)$ is given by Theorem 1. In Section IV new asymptotic random coding bounds on the rate of separable codes for the $B-\mathrm{MAC}$ and the $A-\mathrm{MAC}$ are presented by Theorem 2 and Theorem 3 respectively. In Section $\mathrm{V}$, we introduce the concept of list-decoding codes for the $A-\mathrm{MAC}$ and obtain an upper bound on the rate of these codes, matching with the known lower bound for the very large alphabet size. Based on a simple connection between list-decoding codes and separable codes, we also derive an upper bound on $R^{(A)}(s, q)$, given by Corollary 1 . Finally, in Appendix, we discuss a natural probabilistic generalization of separable codes and give some random coding bounds on the error exponent of almost separable codes and on the rate of separable codes for any symmetric $f-\mathrm{MAC}$.

In particular, as new results we claim the following.

Theorem 1. For any $s \geq 2$ and $q \geq 2$, the rate of $s$-separable $q$-ary codes for the B-MAC satisfies the inequality

$$
R^{(B)}(s, q) \leq\left\{\begin{array}{l}
\frac{s+1}{2 s} \ln q, \quad \text { if } s \text { is odd. } \\
\frac{s+2}{2(s+1)} \ln q, \quad \text { if } s \text { is even. }
\end{array}\right.
$$

Theorem 2. If $s \geq 2$ is fixed and $q \rightarrow \infty$, then the rate $R^{(B)}(s, q)$ satisfies the asymptotic inequality

$$
R^{(B)}(s, q) \geq \frac{s}{2 s-1} \ln q(1+o(1)) .
$$

Theorem 3. If $s \geq 2$ is fixed and $q \rightarrow \infty$, then the rate $R^{(A)}(s, q)$ satisfies the asymptotic inequality

$$
R^{(A)}(s, q) \geq \frac{2}{s+1} \ln q(1+o(1)) .
$$

Corollary 1. For any $s \geq 2$ and $q \geq 2$, the rate of $s$-separable q-ary codes for the A-MAC satisfies the inequality

$$
R^{(A)}(s, q) \leq \frac{2}{s} \ln q
$$




\section{Statement of the Problem}

\section{A. Notations}

Let $q, N, t, s$ and $L$ be integers, where $q \geq 2, N \geq 2,2 \leq s<t / 2,1 \leq L \leq t-s$; symbol $\triangleq$ is the equality by definition; $\mathcal{A}_{q} \triangleq\{0,1, \ldots, q-1\}$ is the standard $q$-ary alphabet; $[N] \triangleq\{1,2, \ldots, N\}$ is the set of integers from 1 to $N ;|A|$ is the size of the set $A ;\lceil b\rceil$ is the least integer $\geq b ;\lfloor b\rfloor$ is the largest integer $\leq b$. A $q$-ary $(N \times t)$-matrix $X=\left(x_{i}(j)\right), i \in[N], j \in[t], x_{i}(j) \in \mathcal{A}_{q}$, with $t$ columns (codewords) $\boldsymbol{x}(j) \triangleq\left(x_{1}(j), \ldots, x_{N}(j)\right) \in \mathcal{A}_{q}^{N}, j \in[t]$, and $N$ rows $\boldsymbol{x}_{i} \triangleq\left(x_{i}(1), x_{i}(2) \ldots, x_{i}(t)\right) \in \mathcal{A}_{q}^{t}, i \in[N]$, is called a q-ary code of length $N$ and size $t$.

For a $q$-ary vector $\boldsymbol{x}=\left(x_{1}, \ldots, x_{s}\right) \triangleq x_{1}^{s} \in \mathcal{A}_{q}^{s}$, define the integer vector $\left(s_{0}, s_{1}, \ldots, s_{q-1}\right)$ of length $q$, where $s_{i}=s_{i}(\boldsymbol{x})$, $0 \leq s_{i} \leq s, i \in \mathcal{A}_{q}$, is the number of positions $i, i \in[s]$, such that $x_{i}=a$. Obviously, $\sum_{i=0}^{q-1} s_{i}=s$. The vector $\left(s_{0}, \ldots, s_{q-1}\right)$ is said to be a type of the $q$-ary vector $x_{1}^{s} \in \mathcal{A}_{q}^{s}$ or, briefly,

$$
T\left(x_{1}^{s}\right) \triangleq\left(s_{0}, \ldots, s_{q-1}\right) .
$$

The set $2^{Y}$ of all subsets of a set $Y$ (or the power set of $Y$ ) is abbreviated by $\mathcal{P}(Y)$. Let $\mathcal{P}(Y, N)$ stand for the Cartesian product of $N$ copies of $\mathcal{P}(Y)$. The union of the $q$-ary vector $x_{1}^{s} \in \mathcal{A}_{q}$ is denoted by

$$
U\left(x_{1}^{s}\right) \triangleq \bigcup_{i \in s} x_{i} \in \mathcal{P}\left(\mathcal{A}_{q}\right)
$$

Let the standard symbol $\left(\begin{array}{c}{[t]} \\ s\end{array}\right)$ be the set of all $s$-subsets of the set $[t]$. For any $\mathbf{e}=\left\{e_{1}, \ldots, e_{s}\right\} \in\left(\begin{array}{c}{[t]} \\ s\end{array}\right)$, called a message, and a code $X$, consider the non-ordered s-collection of codewords

$$
\boldsymbol{x}(\mathbf{e}) \triangleq\left\{\boldsymbol{x}\left(e_{1}\right), \ldots, \boldsymbol{x}\left(e_{s}\right)\right\} .
$$

For a collection of codewords $V=\left\{\boldsymbol{x}\left(i_{1}\right), \ldots, \boldsymbol{x}\left(i_{s}\right)\right\} \subset \mathcal{A}_{q}^{N}$, by $T(V)$ and $U(V)$ we abbreviate the $q$-ary $(N \times q)$ matrix and the vector from $\mathcal{P}\left(\mathcal{A}_{q}, N\right)$ which are defined in the following way

$$
\begin{gathered}
T(V) \triangleq\left(T\left(x_{1}\left(i_{1}\right), \ldots, x_{1}\left(i_{s}\right)\right), \ldots, T\left(x_{N}\left(i_{1}\right), \ldots, x_{N}\left(i_{s}\right)\right)\right)^{T}, \\
U(V) \triangleq\left(U\left(x_{1}\left(i_{1}\right), \ldots, x_{1}\left(i_{s}\right)\right), \ldots, U\left(x_{N}\left(i_{1}\right), \ldots, x_{N}\left(i_{s}\right)\right)\right)^{T} .
\end{gathered}
$$

\section{B. The Symmetric Multiple-Access Channel}

We use the terminology of the noiseless (deterministic) multiple-access channel (MAC), which has $s$ inputs and one output [2]. Let all $s$ input alphabets of MAC be the same and coincide with the alphabet $\mathcal{A}_{q}$. Denote by $Z$ the finite output alphabet of size $|Z|$. Given $s$ inputs $\left(x_{1}, \ldots, x_{s}\right) \in \mathcal{A}_{q}^{s}$ of MAC, the noiseless MAC is prescribed by the function

$$
z=f\left(x_{1}, \ldots, x_{s}\right) \triangleq f\left(x_{1}^{s}\right), \quad z \in Z, x_{1}^{s} \in \mathcal{A}_{q}^{s} .
$$

The deterministic model of MAC is called an $f$-MAC.

Definition 1. An $f$-MAC, given by (9), is said to be the symmetric $f$-MAC if for any permutation $\pi \in S_{s}$, where $S_{s}$ is the symmetric group on $s$ elements, the following equality holds

$$
f\left(x_{1}, \ldots, x_{s}\right)=f\left(x_{\pi(1)}, \ldots, x_{\pi(s)}\right) .
$$

Remark 1. Note that to determine a function $f=f\left(x_{1}, \ldots, x_{s}\right)=f\left(x_{1}^{s}\right)$ for the symmetric $f$-MAC it is necessary and sufficient to define $f$ only on different compositions $\left(s_{0}, s_{1}, \ldots, s_{q}\right)=T\left(x_{1}^{s}\right), x_{1}^{s} \in \mathcal{A}_{q}^{s}$, or in other terms on multisets of cardinality $s$ (s-collections) over $A_{q}$.

In what follows, we consider symmetric $f$-MACs only.

\section{Separable Codes}

For any message $\mathbf{e} \in\left(\begin{array}{c}{[t]} \\ s\end{array}\right)$ and a code $X$, let $\boldsymbol{x}_{i}(\mathbf{e})=\left\{x_{i}\left(e_{1}\right), \ldots, x_{i}\left(e_{s}\right)\right\}, i \in[N]$, be the $s$-collection of signals $(77$ at $s$ symmetric $f$-MAC inputs at the $i$-th time unit. Then the signal $z_{i}, z_{i} \in Z, i \in[N]$, at the output of the symmetric $f$-MAC at the $i$-th time unit is

$$
z_{i}=z_{i}^{(f)}(\mathbf{e}, X) \triangleq f\left(x_{i}\left(e_{1}\right), \ldots, x_{i}\left(e_{s}\right)\right) \in Z .
$$

On the base of the code $X$ and $N$ signals

$$
\boldsymbol{z}^{(f)}(\mathbf{e}, X) \triangleq\left(z_{1}^{(f)}(\mathbf{e}, X), \ldots, z_{N}^{(f)}(\mathbf{e}, X)\right) \in Z^{N},
$$

which are known at the output of MAC, an observer makes the brute force decision about the unknown message e. To identify $\mathbf{e}$, a code $X$ is assigned.

Definition 2. A $q$-ary code $X$ is said to be a s-separable code of size $t$ and length $N$ for the $f$-MAC if all $z^{(f)}(\mathbf{e}, X)$, $\mathbf{e} \in\left(\begin{array}{c}{[t]} \\ s\end{array}\right)$, are distinct. 
Let $t^{(f)}(s, q, N)$ be the maximal size of $s$-separable $q$-ary codes of length $N$ for the $f$-MAC. For fixed $s \geq 2$ and $q \geq 2$, the number

$$
R^{(f)}(s, q) \triangleq \varlimsup_{N \rightarrow \infty} \frac{\ln t^{(f)}(s, q, N)}{N},
$$

is said to be a rate of $s$-separable $q$-ary codes for the $f$-MAC.

\section{Examples of the Symmetric MAC}

1) $A-M A C$ : The $A-$ MAC is described by the function

$$
z=f\left(x_{1}^{s}\right) \triangleq U\left(x_{1}^{s}\right) \subseteq \mathcal{A}_{q},
$$

where the union function $U(\cdot)$ is given in (6). For instance, if $s=4$ and $q=3$, then

$$
U(0,0,1,1)=\{0,1\}, \quad U(1,1,0,2)=\{0,1,2\} .
$$

The cardinality $|Z|$ of output alphabet $Z$ for the $A-\mathrm{MAC}$ is $|Z|=\sum_{k=1}^{\min (s, q)}\left(\begin{array}{c}q \\ k\end{array}\right)$. For $s \geq q$, we have $|Z|=2^{q}-1$.

2) $B-M A C$ : The $B-\mathrm{MAC}$ known also as the compositional channel is described by the function

$$
z=f\left(x_{1}^{s}\right) \triangleq T\left(x_{1}^{s}\right), \quad x_{1}^{s}=\left(x_{1}, \ldots, x_{s}\right) \in \mathcal{A}_{q}^{s},
$$

where the type $T(\cdot)$ of a vector is defined by 5 . For instance, if $s=4$ and $q=3$, then

$$
T(0,0,1,1)=(2,2,0), \quad T(1,1,0,2)=(1,2,1) .
$$

The cardinality of the output alphabet for the $B-\mathrm{MAC}$ is $|Z|=\left(\begin{array}{c}q+s-1 \\ s\end{array}\right), s \geq 2, q \geq 2$. We acknowledge the paper [1], in which the significant applications of the $B-\mathrm{MAC}$ and the $A-\mathrm{MAC}$ were firstly developed. We also refer the reader to [1], [4], [21]-[23], where the maximal output entropy of the $A$-MAC and the $B$-MAC was investigated in different asymptotic and non-asymptotic cases.

3) Erasure MAC: A $q$-ary $f$-MAC is said to be the erasure MAC (briefly, eras-MAC) if it has the $(q+1)$-ary output alphabet $Z \triangleq\{0,1, \ldots, q-1, *\}$ and the output function $z=f\left(x_{1}^{s}\right)$ has the form:

$$
z=f\left(x_{1}, \ldots, x_{s}\right) \triangleq \begin{cases}x, & \text { if } x_{1}=\ldots=x_{s}=x, x \in \mathcal{A}_{q}, \\ *, & \text { otherwise. }\end{cases}
$$

The eras-MAC model can be considered as an adequate description for the transmission of $q$-ary symbols based on the frequency modulation method.

4) Threshold MAC: The threshold $f_{\ell}-\mathrm{MAC}$ (briefly, $\ell$-thr-MAC) has the binary input (i.e., $q=2$ ) and the output alphabet $Z \triangleq \mathcal{A}_{2}=\{0,1\}$, and

$$
z=f_{\ell}\left(x_{1}, \ldots, x_{s}\right) \triangleq \begin{cases}0, & \text { if } \sum_{i=1}^{s} x_{i}<\ell \\ 1, & \text { otherwise }\end{cases}
$$

where terms of the sum are considered as 0 and 1 elements of the ring $\mathbb{Z}$. Separable codes for the $\ell$-thr-MAC can be used in compressed genotyping [24] models in molecular biology.

5) Disjunctive MAC: The disjunctive MAC (briefly, disj-MAC) has the binary input alphabet and the output alphabet $Z \triangleq \mathcal{A}_{2}=\{0,1\}$, and

$$
z=f\left(x_{1}, \ldots, x_{s}\right) \triangleq \begin{cases}0, & \text { if } x_{1}=\ldots=x_{s}=0 \\ 1, & \text { otherwise. }\end{cases}
$$

Notice that the disj-MAC is equivalent to the 1-thr-MAC. The disj-MAC model is interpreted as the transmission of binary symbols based on the impulse modulation method. In addition, the binary $s$-separable codes for the disj-MAC are closely connected with the combinatorial search theory [25] and the information-theoretic model called the design of screening experiments [26].

In what follows, we omit symbol $q=2$ in notations if the corresponding channel is defined only for the binary case.

\section{IMPROVEMENTS OF THE ENTROPY BOUND}

In this section, we first give a general statement called the entropy bound on the rate of separable codes for any symmetric MAC. For an asymptotic regime $s \rightarrow \infty$, we recall the best known bounds on the rate of separable codes for the disjunctive, the erasure, the threshold, the $A$ and the $B$ MACs in Sections III-B III-F, respectively. Finally, in Section III-G, we present Theorem 1, a novel upper bound, which holds for any symmetric MAC and improves the entropy bound. 


\section{A. The Entropy Upper Bound on $R^{(f)}(s, q)$}

Let $\boldsymbol{p}$ be a fixed probability distribution on the alphabet $\mathcal{A}_{q}$ and the vector $\xi_{1}^{s} \triangleq\left\{\xi_{1}, \ldots, \xi_{s}\right\}, \xi_{1}^{s} \in \mathcal{A}_{q}^{s}$, is the $s$-collection of independent random variables having the same distribution, i.e., $\operatorname{Pr}\left\{\xi_{k}=a\right\} \triangleq \boldsymbol{p}(a), k \in[s], a \in \mathcal{A}_{q}$. Introduce the corresponding Shannon entropy of the output of the symmetric $f$-MAC, i.e,

$$
H_{p}^{(f)}(s, q) \triangleq \sum_{z \in Z} \operatorname{Pr}\left\{f\left(\xi_{1}^{s}\right)=z\right\} \cdot \ln \frac{1}{\operatorname{Pr}\left\{f\left(\xi_{1}^{s}\right)=z\right\}} .
$$

The following statement called the entropy upper bound is a conventional information-theoretic bound.

Proposition 1. 27]. The rate $R^{(f)}(s, q)$ of s-separable q-ary codes for the symmetric $f$-MAC satisfies the inequality

$$
R^{(f)}(s, q) \leq C^{(f)}(s, q) \triangleq \frac{\max _{\boldsymbol{p}} H_{\boldsymbol{p}}^{(f)}(s, q)}{s} .
$$

Hereinafter, the value $C^{(f)}(s, q)$ is said to be a capacity of $s$-separable $q$-ary codes for the $f$-MAC.

\section{B. Bounds on the Rate $R^{(d i s j)}(s)$ for the Disjunctive MAC}

One can check [28] that the capacity of $s$-separable binary codes for the $d i s j-\mathrm{MAC}$ is $C^{(d i s j)}(s)=\ln 2 / s$ and the maximum in the right-hand side of 18 is attained at the distribution $\boldsymbol{p}$ with probabilities $\boldsymbol{p}(0)=2^{-1 / s}$ and $\boldsymbol{p}(1)=1-2^{-1 / s}$. The significant results, improving the corresponding entropy $R^{(d i s j)}(s) \leq \ln 2 / s$, were obtained in [29] for $s=2$ and in [30] for $s \geq 11$. In addition, we refer to the best known asymptotic $(s \rightarrow \infty)$ lower [26] and upper [30] bounds on the rate $R^{(d i s j)}(s)$ :

$$
\frac{2(\ln 2)^{2}}{s^{2}}(1+o(1)) \leq R^{(d i s j)}(s) \leq \frac{4 \ln s}{s^{2}}(1+o(1)) .
$$

where the lower bound is based on Proposition 5 formulated in Appendix.

\section{Bounds on the Rate $R^{(\text {eras })}(s, q)$ for the Erasure MAC}

If $q=2$ and $s \rightarrow \infty$, then it is not difficult to establish [31] that the capacity of separable $(s, 2)$-codes for the eras-MAC is $C^{(\text {eras })}(s, 2) \sim \ln 2 / s$ and the maximum in the right-hand side of 18 is asymptotically attained at distribution $\boldsymbol{p}$ with $\boldsymbol{p}(1) \sim \ln 2 / s$ or with $\boldsymbol{p}(0) \sim \ln 2 / s$. In addition, we mention the best known asymptotic $(s \rightarrow \infty)$ lower [32] and upper [26] bounds on the rate $R^{(\text {eras })}(s, 2)$ :

$$
\frac{2(\ln 2)^{2}}{s^{2}}(1+o(1)) \leq R^{(\text {eras })}(s, 2) \leq \frac{4 \ln s}{s^{2}}(1+o(1)) .
$$

Open Problem. In the general case $s \geq 2$ and $q \geq 2$, we conjecture that the capacity $C^{(e r a s)}(s, q)$ of the eras-MAC does not depend on $q \geq 2$, i.e., $C^{(\text {eras })}(s, q)=C^{(\text {eras })}(s, 2)$.

\section{Bounds on the Rate $R^{(\ell-t h r)}(s)$ for the Threshold MAC}

The best known asymptotic $(\ell \geq 2$ is fixed and $s \rightarrow \infty)$ lower and upper bounds on the rate $R^{(\ell-t h r)}(s)$ were presented in [33], [34]:

$$
\frac{\ell^{\ell} e^{-2 \ell}}{(\ell-1) ! 2^{\ell+1} s^{2}}(1+o(1)) \leq R^{(\ell-t h r)}(s) \leq \frac{2 \ell^{2} \ln s}{s^{2}}(1+o(1))
$$

\section{E. Bounds on the Rate $R^{(A)}(s)$ for the $A-M A C$}

For fixed $q$ and $s \rightarrow \infty$, the best known upper bounds on the rate $R^{(A)}(s)$ are based on the upper bound for $R^{(d i s j)}(s)$ and improve the entropy bound. The asymptotic $(s \rightarrow \infty)$ lower and upper bounds were established in [11], [14]

$$
\frac{q-1}{e \log _{2} q} \frac{1}{s^{2}}(1+o(1)) \leq R^{(A)}(s) \leq \frac{2(q-1)}{\log _{2} q} \frac{\ln s}{s^{2}}(1+o(1)) .
$$

F. Bounds on the Rate $R^{(B)}(s)$ for the B-MAC

For fixed $q$ and $s \rightarrow \infty$, the best known lower and upper bounds on the rate $R^{(B)}(s)$ were given in [35], [36] (case $q=2$ ) and in [4] (case $q>2$ )

$$
\frac{(q-1) \ln s}{4 s}(1+o(1)) \leq R^{(B)}(s) \leq \frac{(q-1) \ln s}{2 s}(1+o(1)) .
$$

It is worth to note that the upper bound is actually the entropy bound, and it is quite interesting and challenging to improve it. 


\section{G. Combinatorial Upper Bound for the Symmetric MAC}

In the following theorem, we establish a combinatorial upper bound on the rate of $s$-separable $q$-ary codes for any symmetric $f-$ MAC.

Theorem 1. For any symmetric $f-M A C$ and integers $s \geq 2$ and $q \geq 2$, the rate

$$
R^{(f)}(s, q) \stackrel{(a)}{\leq} R^{(B)}(s, q) \leq\left\{\begin{array}{l}
\frac{s+1}{2 s} \ln q, \quad \text { if } s \text { is } \text { odd } . \\
\frac{s+2}{2(s+1)} \ln q, \quad \text { if } s \text { is even. }
\end{array}\right.
$$

Observe that inequality $(a)$ is evidently implied by Remark 1 Indeed, a separable code for any symmetric $f$-MAC is also a separable code for the $B$-MAC. The maximal output entropy for the $B-\mathrm{MAC}$ was established in [37], and it is known [1] that the capacity of $s$-separable $q$-ary codes for the $B$-MAC is

$$
C^{(B)}(s, q)=\sum_{s_{0}+\ldots+s_{q-1}=s} \frac{s !}{s_{0} ! \ldots s_{q-1} !} \frac{1}{q^{s}} \ln \left(\frac{s_{0} ! \ldots s_{q-1} !}{s ! / q^{s}}\right) .
$$

Therefore, $C^{(B)}(s, q) \sim \ln q$ as $q \rightarrow \infty$, and Theorem 1 improves the entropy upper bound 18 for the $B$-MAC.

Proof of Theorem 1] Fix an arbitrary $q$-ary $(N \times t)$-code $X$. For any $\alpha, 0<\alpha<1$, without loss of generality, we may assume that all codewords from $X$ are distinct and the length $N$ can be represented as a sum of two integers $\alpha N$ and $(1-\alpha) N$. Given $X$, introduce the bipartite graph

$$
G=G(X)=(V, E) \triangleq\left(V_{1} \cup V_{2}, E\right), \quad\left|V_{1}\right|=q^{\alpha N},\left|V_{2}\right|=q^{(1-\alpha) N},
$$

defined as follows. Let the vertices in $V_{1}$ and $V_{2}$ correspond to distinct $q$-ary vectors of length $\alpha N$ and $(1-\alpha) N$, respectively. Two vertices $v_{1} \in V_{1}$ and $v_{2} \in V_{2}$ are connected with an edge if and only if the code $X$ contains a codeword of length $N=\alpha N+(1-\alpha) N$ which is the concatenation of two $q$-ary vectors corresponding to $v_{1}$ and $v_{2}$. Thus, we obtain the graph $G(X)$ having $|V|=q^{(1-\alpha) N}+q^{\alpha N}$ vertices and $t$ edges, identified by the indexes $[t]$ of the code $X$. In addition, any message $\mathbf{e} \in\left(\begin{array}{c}{[t]} \\ s\end{array}\right)$ is interpreted as a non-ordered $s$-collection of edges.

Let $X$ be a $q$-ary $s$-separable code for the $f$-MAC. Suppose, seeking a contradiction, that there exists a simple cycle $C_{2 \ell}$ of length $2 \ell \leq 2 s$ in $G(X)$. Enumerate edges in $C_{2 \ell}$ by $e_{1}, \ldots, e_{2 \ell}$, where $e_{i}$ and $e_{i+1}$ are adjacent for any $i \in[2 \ell-1]\left(e_{1}\right.$ and $e_{2 \ell}$ are also adjacent). Define the set $E_{1}$ as $\left\{e_{1}, e_{3}, \ldots, e_{2 \ell-1}\right\}$, and let $E_{2}$ be the remaining edges of the cycle. Consider an arbitrary subset $\mathcal{S} \subset[t] \backslash\left\{E_{1} \cup E_{2}\right\}$ of the size $|\mathcal{S}|=s-\ell$ and define two messages $\mathbf{e}_{i} \triangleq E_{i} \cup \mathcal{S} \in\left(\begin{array}{c}{[t]} \\ s\end{array}\right), i=1,2$. It is easy to check that outputs of the symmetric $f$-MAC for these messages are the same, i.e., $\boldsymbol{z}^{(f)}\left(\mathbf{e}_{1}, X\right)=\boldsymbol{z}^{(f)}\left(\mathbf{e}_{2}, X\right)$. This contradicts to Definition 2

It is known (e.g., see [38]) that if a bipartite graph with two parts of sizes $n$ and $m$ does not contain any simple cycle of length $\leq 2 s$, then the number $t$ of its edges is

$$
t \leq \begin{cases}(2 s-3)\left[(m n)^{\frac{s+1}{2 s}}+m+n\right], & \text { if } s \text { is odd } \\ (2 s-3)\left[m^{\frac{s+2}{2 s}} n^{1 / 2}+m+n\right], & \text { if } s \text { is even }\end{cases}
$$

For odd $s$, we obtain

$$
t \leq(2 s-3)\left[q^{N \frac{s+1}{2 s}}+q^{\alpha N}+q^{(1-\alpha) N}\right] \leq 3(2 s-3) q^{N \max \left\{\frac{s+1}{2 s}, \alpha,(1-\alpha)\right\}}
$$

Taking $\alpha=1 / 2$, we derive

$$
t \leq 3(2 s-3) q^{\frac{s+1}{2 s} N},
$$

and the rate 13 is upper bounded as in $(19)$. Applying the second inequality for even $s$, we have

$$
t \leq(2 s-3)\left[q^{\frac{N}{2}\left(1+\frac{2 \alpha}{s}\right)}+q^{\alpha N}+q^{(1-\alpha) N}\right] \leq 3(2 s-3) q^{N \max \left\{\frac{s+2 \alpha}{2 s}, \alpha, 1-\alpha\right\}} .
$$

Taking $\alpha$ as a root of inequality $\frac{s+2 \alpha}{2 s}=1-\alpha$, i.e., $\alpha=\frac{s}{2(s+1)}$, we obtain

$$
t \leq 3(2 s-3) q^{\frac{s+2}{2(s+1)} N},
$$

i.e., the rate (13) satisfies 19 .

\section{ASYMPTOTIC RANDOM CODING BOUNDS FOR THE $A-$ MAC AND THE $B-$ MAC}

In this section, we apply the probabilistic method to construct asymptotic lower bounds on the rate of $s$-separable $q$-ary codes for the $A-\mathrm{MAC}$ and the $B-\mathrm{MAC}$. 


\section{A. Random Coding Lower Bound on $R^{(B)}(s, q)$}

An asymptotic $(q \rightarrow \infty)$ random coding lower bound on the rate of $s$-separable $q$-ary codes for the $B$-MAC is given by

Theorem 2. If $s \geq 2$ is fixed and $q \rightarrow \infty$, then the rate $R^{(B)}(s, q)$ satisfies the asymptotic inequality

$$
R^{(B)}(s, q) \geq \frac{s}{2 s-1} \ln q(1+o(1)) .
$$

Proof of Theorem 2. Consider the ensemble of matrices $X=\left(x_{i}(j)\right)$, where entries $x_{i}(j), i \in[N], j \in[t]$, are chosen independently and equiprobable from the alphabet $\mathcal{A}_{q}$. Define a $b a d$ event $B_{j}$ : "there exist two distinct messages e $\neq \hat{\mathbf{e}}$ from $\left(\begin{array}{c}{[t]} \\ s\end{array}\right)$ so that $j \in \mathbf{e}, j \notin \hat{\mathbf{e}}$ and $T(\boldsymbol{x}(\mathbf{e}))=T(\boldsymbol{x}(\hat{\mathbf{e}}))$ ", where the matrix $T(\cdot)$ is defined by (8). To establish the existence of an $s$-separable $q$-ary code for the $B$-MAC, we shall upper bound the probability of the bad event

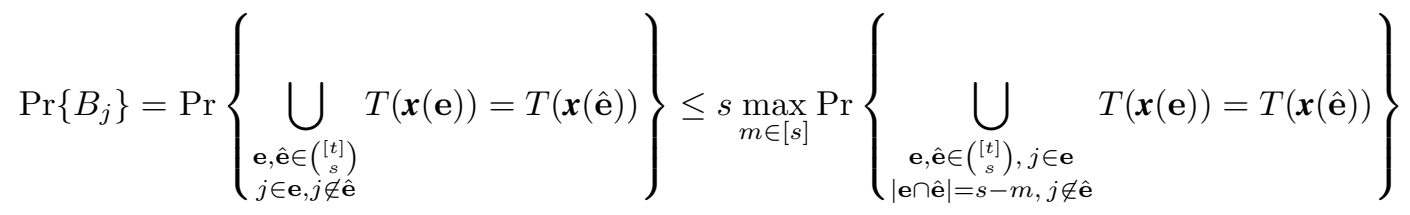

$$
\begin{aligned}
& \leq s \max _{m \in[s]} t^{2 m-1} \operatorname{Pr}\left\{\begin{array}{c}
T(\boldsymbol{x}(\mathbf{e}))=T(\boldsymbol{x}(\hat{\mathbf{e}}))) \\
\mathbf{e}, \hat{\mathbf{e}} \in\left(\begin{array}{c}
{[t]} \\
\mid \mathbf{e}) \\
|\mathbf{e} \hat{\mathbf{e}}|=s-m
\end{array}\right.
\end{array}\right\}=s \max _{m \in[s]} t^{2 m-1}\left(\operatorname{Pr}\left\{T\left(u_{1}, \ldots, u_{m}\right)=T\left(v_{1}, \ldots, v_{m}\right)\right\}\right)^{N} \text {, }
\end{aligned}
$$

where the first and the second inequalities are evident consequences of the union bound, and $\left.\left\{u_{i}, v_{i}\right\}\right|_{i=1} ^{m}$ are independent random variables having the uniform distribution on the set $\mathcal{A}_{q}$. Let us estimate the probability that two random $m$-tuples have the same type

$$
\operatorname{Pr}\left\{T\left(u_{1}^{m}\right)=T\left(v_{1}^{m}\right)\right\}=\operatorname{Pr}\left\{\bigcup_{\pi \in S_{m}}\left[\bigcap_{k=1}^{m}\left(u_{k}=v_{\pi(i)}\right)\right]\right\} \leq m ! \cdot \operatorname{Pr}\left\{\bigcap_{k=1}^{m}\left(u_{k}=v_{\pi(k)}\right)\right\}=\frac{m !}{q^{m}} .
$$

Therefore,

$$
\operatorname{Pr}\left\{B_{j}\right\} \leq s \max _{m \in[s]}\left[t^{2 m-1}\left(m ! / q^{m}\right)^{N}\right]
$$

Since $\operatorname{Pr}\left\{B_{j}\right\}$ does not depend on $j \in[t]$, we deduce that if the upper bound given above is less than $1 / 2$, then there exists an $s$-separable $q$-ary code for the $B$-MAC of size $t / 2$ and length $N$. Thus, the lower bound on $R^{(B)}(s, q)$ is as follows

$$
R^{(B)}(s, q) \geq \min _{m \in[s]}\left[\frac{m \ln q-\ln m !}{2 m-1}\right] \text {. }
$$

This leads to the statement of Theorem 2 .

\section{B. Random Coding Lower Bound on $R^{(A)}(s, q)$}

Now we establish an asymptotic random coding lower bound on the rate of $s$-separable $q$-ary codes for the $A$-MAC which is presented by

Theorem 3. If $s \geq 2$ is fixed and $q \rightarrow \infty$, then the rate $R^{(A)}(s, q)$ satisfies the asymptotic inequality

$$
R^{(A)}(s, q) \geq \frac{2}{s+1} \ln q(1+o(1))
$$

Proof of Theorem 3. Consider the ensemble of matrices $X=\left(x_{i}(j)\right)$, where entries $x_{i}(j), i \in[N], j \in[t]$, are chosen independently and equiprobable from the alphabet $\mathcal{A}_{q}$. Define a $b a d$ event $A_{j}$ : "there exist two distinct messages e $\neq \hat{\mathbf{e}}$ from $\left(\begin{array}{c}{[t]} \\ s\end{array}\right)$ so that $j \in \mathbf{e}, j \notin \hat{\mathbf{e}}$ and $U(\boldsymbol{x}(\mathbf{e}))=U(\boldsymbol{x}(\hat{\mathbf{e}}))$ ", where the vector $U(\cdot) \in \mathcal{P}\left(\mathcal{A}_{q}, N\right)$ is defined by (8). To establish the existence of an $s$-separable $q$-ary code for the $A$-MAC, we shall upper bound the probability of the bad event

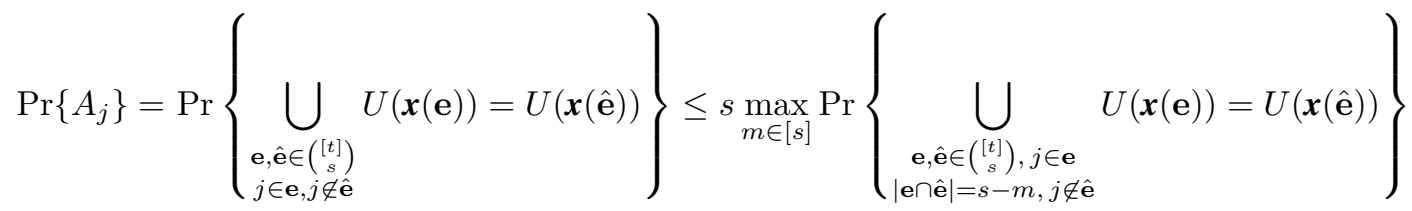

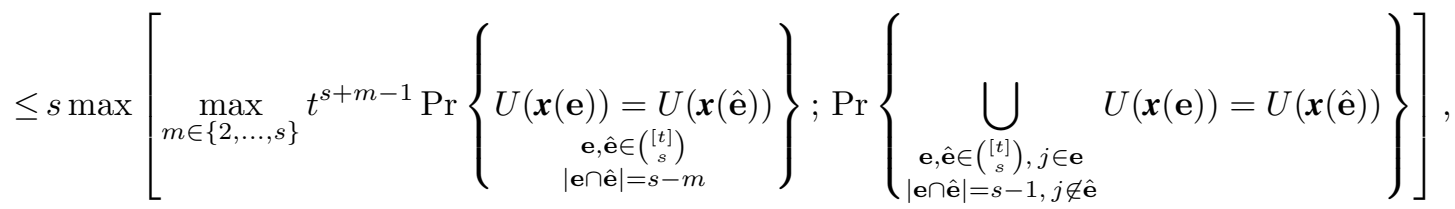


where the first and the second inequalities are evident consequences of the union bound. For any e, $\hat{\mathbf{e}} \in\left(\begin{array}{ll}{[t]} \\ s\end{array}\right),|\mathbf{e} \cap \hat{\mathbf{e}}|=s-m$, let us estimate the probability $\operatorname{Pr}\{U(\boldsymbol{x}(\mathbf{e}))=U(\boldsymbol{x}(\hat{\mathbf{e}}))$ as follows

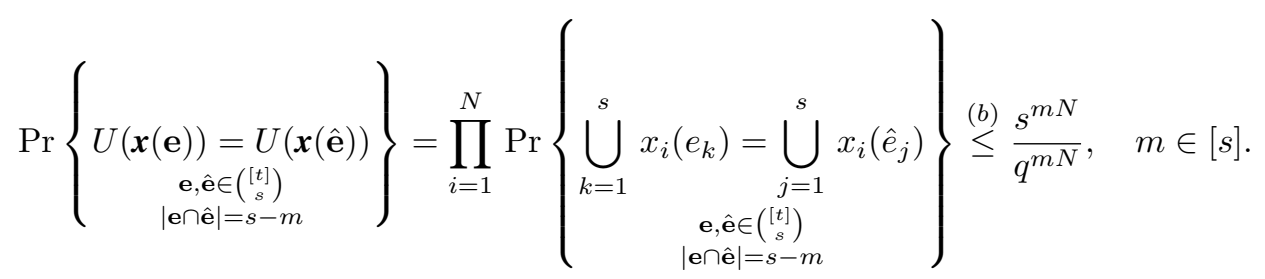

To prove $(b)$ in the last inequality, we employ the following fact. If $\xi_{1}, \ldots, \xi_{m+s}$ are independent and distributed uniformly over $\mathcal{A}_{q}$, then

$$
\operatorname{Pr}\left\{\bigcup_{k=1}^{s} \xi_{k}=\bigcup_{j=m+1}^{m+s} \xi_{j}\right\} \leq \operatorname{Pr}\left\{\bigcup_{k=1}^{m} \xi_{k} \subset \bigcup_{i=m+1}^{m+s} \xi_{i}\right\} \leq\left(\operatorname{Pr}\left\{\xi_{1} \in \bigcup_{i=m+1}^{m+s} \xi_{i}\right\}\right)^{m} \leq \frac{s^{m}}{q^{m}}
$$

For the second probability under the maximum in 20, we obtain an upper bound in a different way. Let $E_{j}$ consist of all possible pairs $(\mathbf{e}, \hat{\mathbf{e}})$ so that $\mathbf{e}, \hat{\mathbf{e}} \in\left(\begin{array}{c}{[t]} \\ s\end{array}\right), j \in \mathbf{e}, j \notin \hat{\mathbf{e}}$ and $|\mathbf{e} \cap \hat{\mathbf{e}}|=s-1$. Since $|\mathbf{e} \cap \hat{\mathbf{e}}|=s-1$, there exists $\hat{j} \in[t]$ such that $\mathbf{e}=\{j\} \cup\{\mathbf{e} \cap \hat{\mathbf{e}}\}$ and $\hat{\mathbf{e}}=\{\hat{j}\} \cup\{\mathbf{e} \cap \hat{\mathbf{e}}\}$. For a real parameter $a, 0<a<1$, we represent the event $\{U(\boldsymbol{x}(\mathbf{e}))=U(\boldsymbol{x}(\hat{\mathbf{e}}))\}$ as a disjoint union of two events. For the first one, we additionally require the Hamming distance $d_{H}(\cdot)$ between $\boldsymbol{x}(j)$ and $\boldsymbol{x}(\hat{j})$ to be at least $a N$, i.e., $A_{j}(\mathbf{e}, \hat{\mathbf{e}}, \geq a) \triangleq\left\{U(\boldsymbol{x}(\mathbf{e}))=U(\boldsymbol{x}(\hat{\mathbf{e}})), d_{H}(\boldsymbol{x}(j), \boldsymbol{x}(\hat{j})) \geq a N\right\}$. The remaining one is $A_{j}(\mathbf{e}, \hat{\mathbf{e}},<a) \triangleq\left\{U(\boldsymbol{x}(\mathbf{e}))=U(\boldsymbol{x}(\hat{\mathbf{e}})), d_{H}(\boldsymbol{x}(j), \boldsymbol{x}(\hat{j}))<a N\right\}$. Then we deal with each event individually. More concretely,

$$
\begin{aligned}
\operatorname{Pr}\left\{\bigcup_{(\mathbf{e}, \hat{\mathbf{e}}) \in E_{j}} U(\boldsymbol{x}(\mathbf{e}))=U(\boldsymbol{x}(\hat{\mathbf{e}})\}\right. & =\operatorname{Pr}\left\{\bigcup_{(\mathbf{e}, \hat{\mathbf{e}}) \in E_{j}} A_{j}(\mathbf{e}, \hat{\mathbf{e}}, \geq a)\right\}+\operatorname{Pr}\left\{\bigcup_{(\mathbf{e}, \hat{\mathbf{e}}) \in E_{j}} A_{j}(\mathbf{e}, \hat{\mathbf{e}},<a)\right\} \\
& \leq t^{s} \operatorname{Pr}\left\{\begin{array}{c}
A_{j}(\mathbf{e}, \hat{\mathbf{e}}, \geq a) \\
(\mathbf{e}, \hat{\mathbf{e}}) \in E_{j}
\end{array}\right\}+t \operatorname{Pr}\left\{d_{H}(\boldsymbol{x}(j), \boldsymbol{x}(\hat{j}))<a N\right\},
\end{aligned}
$$

where the inequality is implied by the union bound, and $\hat{j} \in[t], \hat{j} \neq j$. Let us estimate the probability that two random $q$-ary vectors of length $N$ have the Hamming distance at most $a N$

$$
\operatorname{Pr}\left\{d_{H}(\boldsymbol{x}(j), \boldsymbol{x}(\hat{j}))<a N\right\}=\sum_{i=N-\lfloor a N\rfloor}^{N} \operatorname{Pr}\left\{d_{H}(\boldsymbol{x}(j), \boldsymbol{x}(\hat{j}))=N-i\right\}=\sum_{i=N-\lfloor a N\rfloor}^{N}\left(\begin{array}{c}
N \\
i
\end{array}\right)\left(\frac{1}{q}\right)^{i}\left(1-\frac{1}{q}\right)^{N-i}<\frac{2^{N}}{q^{(1-a) N}}
$$

Now, for any $(\mathbf{e}, \hat{\mathbf{e}}) \in E_{j}$, we proceed with the event $A_{j}(\mathbf{e}, \hat{\mathbf{e}}, \geq a)=\left\{U(\boldsymbol{x}(\mathbf{e}))=U(\boldsymbol{x}(\hat{\mathbf{e}})), d_{H}(\boldsymbol{x}(j), \boldsymbol{x}(\hat{j})) \geq a N\right\}$ as follows

$$
\begin{array}{r}
\operatorname{Pr}\left\{A_{j}(\mathbf{e}, \hat{\mathbf{e}}, \geq a)\right\}=\sum_{i=0}^{N-\lceil a N\rceil} \operatorname{Pr}\left\{U(\boldsymbol{x}(\mathbf{e}))=U(\boldsymbol{x}(\hat{\mathbf{e}})) \mid d_{H}(\boldsymbol{x}(j), \boldsymbol{x}(\hat{j}))=N-i\right\} \operatorname{Pr}\left\{d_{H}(\boldsymbol{x}(j), \boldsymbol{x}(\hat{j}))=N-i\right\} \\
\stackrel{(c)}{\leq} \sum_{i=0}^{N-\lceil a N\rceil}\left(\begin{array}{c}
N \\
i
\end{array}\right)\left(\frac{1}{q}\right)^{i}\left(1-\frac{1}{q}\right)^{N-i}\left(\frac{(s-1)^{2}}{q^{2}}\right)^{N-i}<\frac{\left(2 s^{2}\right)^{N}}{q^{(1+a) N}} .
\end{array}
$$

To prove $(c)$ in the last inequality, we use the following fact. If $\xi_{1}, \ldots, \xi_{s+1}$ are independent and distributed uniformly over $\mathcal{A}_{q}$, then

$$
\operatorname{Pr}\left\{\bigcup_{k=1}^{s} \xi_{k}=\bigcup_{j=2}^{s+1} \xi_{j}, \xi_{1} \neq \xi_{s+1}\right\} \leq \operatorname{Pr}\left\{\xi_{1} \in \bigcup_{j=2}^{s} \xi_{j}, \xi_{s+1} \in \bigcup_{j=2}^{s} \xi_{j}\right\} \leq \frac{(s-1)^{2}}{q^{2}}
$$

Therefore,

$$
\operatorname{Pr}\left\{\bigcup_{\{\mathbf{e}, \hat{\mathbf{e}}\} \in E_{j}} U(\boldsymbol{x}(\mathbf{e}))=U(\boldsymbol{x}(\hat{\mathbf{e}}))\right\} \leq \min _{0<a<1}\left[t^{s} \frac{\left(2 s^{2}\right)^{N}}{q^{(1+a) N}}+t \frac{2^{N}}{q^{(1-a) N}}\right] \leq 2 \min _{0<a<1}\left\{\max \left[\frac{t^{s}\left(2 s^{2}\right)^{N}}{q^{(1+a) N}} ; \frac{t 2^{N}}{q^{(1-a) N}}\right]\right\} .
$$

Finally, summarizing the above arguments, we obtain

$$
\operatorname{Pr}\left\{A_{j}\right\} \leq 2 s \max \left[\max _{m \in\{2, \ldots, s\}} \frac{t^{s+m-1} s^{m N}}{q^{m N}} ; \min _{0<a<1}\left\{\max \left[\frac{t^{s}\left(2 s^{2}\right)^{N}}{q^{(1+a) N}} ; \frac{t 2^{N}}{q^{(1-a) N}}\right]\right\}\right] .
$$


Since $\operatorname{Pr}\left\{A_{j}\right\}$ does not depend on $j \in[t]$, we deduce that if the upper bound given above is less than $1 / 2$, then there exists an $s$-separable $q$-ary code for the $A$-MAC of size $t / 2$ and length $N$. Thus, the asymptotic $(q \rightarrow \infty)$ lower bound on $R^{(A)}(s, q)$ is as follows

$$
R^{(A)}(s, q) \geq \min \left[\frac{2}{s+1} ; \max _{0<a<1}\left\{\min \left[\frac{1+a}{s} ; 1-a\right]\right\}\right] \ln q(1+o(1))=\frac{2}{s+1} \ln q(1+o(1)) .
$$

Remark 2. It is worth noticing that if we upper bound the probabilities in 20 for each $m \in[s]$ with the help of $(21)$, then we would get only $R^{(A)}(s, q) \geq \frac{1}{s} \ln q(1+o(1))$ as $q \rightarrow \infty$.

\section{List Decoding Codes for the $A-\mathrm{MAC}$}

After giving definitions and notations, in Section $\mathrm{V}-\mathrm{A}$, we derive several useful properties establishing a connection between list-decoding codes for the $A-\mathrm{MAC}$ and separable codes for the $A-\mathrm{MAC}$ and a relation between list decoding codes over alphabets of different sizes. We recall the best known lower bounds on the rate of list-decoding codes in Section V-B Finally, we present a new combinatorial upper bound on the rate of list-decoding codes in Section V-B. which also leads to an upper bound on the rate of separable codes for the $A-\mathrm{MAC}$.

\section{A. Notations and Definitions}

Recall that $\mathcal{P}\left(\mathcal{A}_{q}, N\right)$ stands for the Cartesian product of $N$ copies of $\mathcal{P}\left(\mathcal{A}_{q}\right)$, where $\mathcal{P}\left(\mathcal{A}_{q}\right)$ is the set of all subsets of $\mathcal{A}_{q}$. A vector $\mathcal{Q}=\left(\mathcal{Q}_{1}, \ldots, \mathcal{Q}_{N}\right)^{T} \in \mathcal{P}\left(\mathcal{A}_{q}, N\right)$ is said to cover a column $\boldsymbol{x}=\left(x_{1}, \ldots, x_{N}\right)^{T} \in \mathcal{A}_{q}^{N}$ if $x_{i} \in \mathcal{Q}_{i}$ for all $i \in[N]$.

Definition 3. [32]. Given integers $s \geq 1$ and $L \geq 1$, a $q$-ary code $X$ of size $t$ and length $N$ is said to be a list-decoding $(s, L, q)$-code of size $t$ and length $N$ if, for any $s$-collection of codewords $\left\{\boldsymbol{x}\left(j_{1}\right), \ldots, \boldsymbol{x}\left(j_{s}\right)\right\}$, the vector $U\left(\boldsymbol{x}\left(j_{1}\right), \ldots, \boldsymbol{x}\left(j_{s}\right)\right)$, defined by (8), covers not more than $L-1$ other codewords of the code $X$.

In the case $s \geq 2$ and $L=1$, the list-decoding $(s, 1, q)$-code (or $s$-frameproof code [9]) is an $(\leq s)$-separable $q$-ary code for the $A$-MAC. Moreover, list-decoding $(s, 1, q)$-code provides a simple factor decoding algorithm, that picks the unknown message $\mathbf{e}=\left(e_{1}, \ldots, e_{s}\right) \in\left(\begin{array}{c}{[t]} \\ s\end{array}\right)$ by searching all codewords of $X$ covered by the output signal

$$
\boldsymbol{z}^{(A)}(\mathbf{e}, X)=U\left(\boldsymbol{x}\left(e_{1}\right), \ldots, \boldsymbol{x}\left(e_{s}\right)\right)=\left(\bigcup_{m=1}^{s} x_{1}\left(e_{m}\right), \ldots, \bigcup_{m=1}^{s} x_{N}\left(e_{m}\right)\right)^{T} .
$$

In the general case $L \geq 1$, the algorithm provides a subset of $[t]$ that contains $s$ elements of the message e and at most $L-1$ extra elements.

Let $t(s, L, q, N)$ be the maximal possible size of list-decoding $(s, L, q)$-codes of length $N$. For fixed $s \geq 2, L \geq 1$ and $q \geq 2$, define a rate of list-decoding $(s, L, q)$-codes:

$$
R(s, L, q) \triangleq \varlimsup_{N \rightarrow \infty} \frac{\ln t(s, L, q, N)}{N} .
$$

An important evident connection between $s$-separable $q$-ary codes for the $A$-MAC and list-decoding $(s, L, q)$-codes is formulated as

Proposition 2. Any s-separable q-ary code for the $A-M A C$ is a list-decoding $(s-1,2, q)$-code and, therefore, the rate of s-separable q-ary code for the A-MAC satisfies the inequality

$$
R^{(A)}(s, q) \leq R(s-1,2, q), \quad s \geq 2, \quad q \geq 2 .
$$

Proposition 2 can be seen as a simple reformulation of the corresponding properties of binary list-decoding superimposed codes firstly introduced in [18]. A nontrivial recurrent inequality for the rate $R(s, L, q)$ of list-decoding $(s, L, q)$-codes is established by

Proposition 3. For any integers $q^{\prime}>q \geq 2, s \geq 2$ and $L \geq 1$ the following inequality holds:

$$
R(s, L, q) \geq \frac{R\left(s, L, q^{\prime}\right)}{\left\lceil q^{\prime} /(q-1)\right\rceil} .
$$

Proof of Proposition 3 Assume that there exists a list-decoding $\left(s, L, q^{\prime}\right)$-code $X^{\prime}$ of length $N$ and size $t$. Let $l \triangleq\left\lceil q^{\prime} /(q-1)\right\rceil$. Consider a $q$-ary code $C$ of length $l$ and size $l(q-1) \geq q^{\prime}$, which is composed from all possible codewords with one nonzero symbol:

$$
\left|\begin{array}{ccccccccc}
1 & 0 & \ldots & 0 & \ldots & q-1 & 0 & \ldots & 0 \\
0 & 1 & \ldots & 0 & \ldots & 0 & q-1 & \ldots & 0 \\
\vdots & \vdots & \ddots & \vdots & \ldots & \vdots & \vdots & \ddots & \vdots \\
0 & 0 & \ldots & 1 & \ldots & 0 & 0 & \ldots & q-1
\end{array}\right|
$$


Let us consider an injective map $\phi: \mathcal{A}_{q^{\prime}} \rightarrow C$ such that $\phi(i)$ is the $(i+1)$ th codeword of $C$. To construct a $q$-ary code $X$ of length $l N$ and size $t$, we replace each symbol $a \in \mathcal{A}_{q^{\prime}}$ in all codewords in $X^{\prime}$ by $q$-ary codeword $\phi(a)$. One can easily check that the code $X$ is a list-decoding $(s, L, q)$-code.

\section{B. Lower Bound on the rate $R(s, L, q)$}

In [32], applying Proposition 3 and random coding arguments, the author established the lower bound on the rate of listdecoding $(s, L, q)$-codes which can be formulated as

Theorem 4. [32, Theorem 2]. 1. For any fixed $q \geq 2, s \geq 2$ and $L \geq 1$ the following lower bound holds:

$$
R(s, L, q) \geq \underline{R}(s, L, q) \triangleq \max _{q^{\prime} \geq q} \frac{-\ln P\left(q^{\prime}, s, L\right)}{(s+L-1) k\left(q, q^{\prime}\right)},
$$

where

$$
\begin{aligned}
P(q, s, L) & \triangleq \sum_{m=1}^{\min (q, s)}\left(\begin{array}{c}
q \\
m
\end{array}\right)\left(\frac{m}{q}\right)^{L} \times \sum_{k=0}^{m}(-1)^{k}\left(\begin{array}{c}
m \\
k
\end{array}\right)\left(\frac{m-k}{q}\right)^{s}, \\
k\left(q, q^{\prime}\right) & \triangleq \begin{cases}1, & \text { for } q=q^{\prime}, \\
\left\lceil\frac{q^{\prime}}{q-1}\right\rceil, & \text { otherwise. }\end{cases}
\end{aligned}
$$

2. For any fixed $q \geq 2, L \geq 1$ and $s \rightarrow \infty$

$$
\underline{R}(s, L, q) \geq \frac{L(q-1)(\ln 2)^{2}}{s^{2}}(1+o(1)), \quad s \rightarrow \infty .
$$

3. For any fixed $s \geq 2, L \geq 1$ and $q \rightarrow \infty$,

$$
\underline{R}(s, L, q)=\frac{L}{s+L-1} \ln q(1+o(1) .
$$

The lower bound $\underline{R}(s, L, q)$ defined by [24]-26] improves the best previously known bounds presented in [11], [15], [31] in

\begin{tabular}{|c|c|c|c|c|c|}
\hline$s$ & 2 & 3 & 4 & 5 & 6 \\
\hline$R(s, 1,2) \geq$ & $0.1438^{1,2,4}$ & $0.0554^{2}$ & $0.0304^{2}$ & $0.0194^{2}$ & $0.0134^{2}$ \\
\hline$q^{\prime}(s, 1,2)$ & 2 & 6 & 7 & 9 & 10 \\
\hline$R(s, 2,2) \geq$ & $0.1703^{2}$ & $0.0799^{2}$ & $0.0474^{2}$ & $0.0316^{2}$ & $0.0226^{2}$ \\
\hline$q^{\prime}(s, 2,2)$ & 2 & 6 & 8 & 9 & 10 \\
\hline$R(s, 1,3) \geq$ & $0.2939^{1,3,4}$ & $0.1171^{1,4}$ & $0.0551^{1}$ & $0.0360^{1}$ & $0.0253^{1}$ \\
\hline$q^{\prime}(s, 1,3)$ & 3 & 3 & 8 & 8 & 10 \\
\hline$R(s, 2,3) \geq$ & $0.3662^{1}$ & $0.1583^{1}$ & $0.0864^{1}$ & $0.0585^{1}$ & $0.0425^{1}$ \\
\hline$q^{\prime}(s, 2,3)$ & 3 & 3 & 8 & 10 & 10 \\
\hline
\end{tabular}
asymptotics ( $q$ is fixed, $s \rightarrow \infty$ ) and in a wide range of parameters $(q, s, L)$ as well. Some numerical results and a comparison of bounds are presented in Table I] where $q^{\prime}(s, L, q)$ denotes the argument of maximum 24.

TABLE I

THE BEST KNOWN LOWER BOUNDS ON $R(s, L, q)$

C. Upper Bounds on the rates $R(s, L, q)$ and $R^{(A)}(s, q)$

It was also conjectured in [32] that the lower bound [28] is tight. We prove the conjecture in

Theorem 5. For any $s \geq 2, L \geq 1$ and $q \geq 2$ the rate $R(s, L, q)$ of list-decoding $(s, L, q)$-codes satisfies the inequality

$$
R(s, L, q) \leq \frac{L}{s+L-1} \ln q .
$$

In particular, Theorem 5 and Proposition 2 yield to the following statement.

Corollary 1. For any $s \geq 2$ and $q \geq 2$, the rate of s-separable $q$-ary codes for the A-MAC satisfies the inequality

$$
R^{(A)}(s, q) \leq \frac{2}{s} \ln q
$$

Proof of Theorem 5 Consider an arbitrary code $X$ of length $N$ and size $t$. For a convenience of the proof, we will use indexes $j(i)$ of codewords (rows) which can exceed $t(N)$, assuming that the indexes are cyclically ordered, i.e.,

$$
x_{n}(j)=x_{n^{\prime}}\left(j^{\prime}\right), \quad \text { for } n-n^{\prime} \equiv 0 \quad \bmod N, \quad j-j^{\prime} \equiv 0 \quad \bmod t .
$$


For a codeword $\boldsymbol{x}(j) \in \mathcal{A}_{q}^{N}, j \in[t]$, by

$$
\boldsymbol{x}_{n}^{n+L-1}(j) \triangleq\left(x_{n}(j), \ldots, x_{n+L-1}(j)\right) \in \mathcal{A}_{q}^{L},
$$

we abbreviate a projection of the codeword $\boldsymbol{x}(j)$ on the coordinates $n, n+1, \ldots, n+L-1$. A codeword $\boldsymbol{x}(j), j \in[t]$, is said to be an $L$-rare in $X$ if there exists a row index $n \in[N]$ such that the number of codeword indexes $j^{\prime} \in[t], j^{\prime} \neq j$, with the same projection $\boldsymbol{x}_{n}^{n+L-1}\left(j^{\prime}\right)=\boldsymbol{x}_{n}^{n+L-1}(j)$ is at most $L-1$. Let $r=r_{L}(X)$ be the number of codewords which are $L$-rare in $X$. For each $L$-rare codeword $\boldsymbol{x}(j)$, we can choose a row index $n \in[N]$, a $q$-ary sequence $\left(a_{1}, \ldots, a_{L}\right) \in \mathcal{A}_{q}^{L}$ and an ordinal number (from 1 to $L$ ) of the $\boldsymbol{x}(j)$ among all $\leq L$ codewords $\boldsymbol{x}\left(j^{\prime}\right), j^{\prime} \in[t]$, for which $\boldsymbol{x}_{n}^{n+L-1}\left(j^{\prime}\right)=\boldsymbol{x}_{n}^{n+L-1}(j)=\left(a_{1}, \ldots, a_{L}\right)$. This correspondence is injective. Therefore, the following claim holds.

Lemma 1. For any code $X$ of length $N$, the number $r_{L}(X)$ of its L-rare codewords satisfies the inequality

$$
r=r_{L}(X) \leq N L q^{L} .
$$

Now we formulate another auxiliary statement.

Lemma 2. If a q-ary code $X$ of length $N$ has a size

$$
t>N L q^{L} \sum_{k=0}^{L-1} k !,
$$

then there exists an ordered set of codewords $\mathcal{L}_{s}=\left(\boldsymbol{x}\left(j_{1}\right), \ldots, \boldsymbol{x}\left(j_{L}\right)\right)$ such that there is no $L$-rare codeword in $\mathcal{L}_{s}$. In addition, for any $k \in[L-1]$, the projections of $\boldsymbol{x}\left(j_{k}\right)$ and $\boldsymbol{x}\left(j_{k+1}\right)$ on the coordinates $1+k(s-1), 2+k(s-1), \ldots, L+k(s-1)$ are the same, i.e.,

$$
\boldsymbol{x}_{1+k(s-1)}^{L+k(s-1)}\left(j_{k}\right)=\boldsymbol{x}_{1+k(s-1)}^{L+k(s-1)}\left(j_{k+1}\right), \quad k \in[L-1] .
$$

Proof of Lemma 2 For any $j_{1} \in[t]$, we shall try to construct a sequence $\mathcal{L}\left(j_{1}\right)=\left(\boldsymbol{x}\left(j_{1}\right), \boldsymbol{x}\left(j_{2}\right), \ldots, \boldsymbol{x}\left(j_{L}\right)\right)$ of $L$ codewords by the following rules. The first element of the sequence $\mathcal{L}\left(j_{1}\right)$ is $\boldsymbol{x}\left(j_{1}\right)$. Let a sequence $\left(\boldsymbol{x}\left(j_{1}\right), \boldsymbol{x}\left(j_{2}\right), \ldots, \boldsymbol{x}\left(j_{k}\right)\right)$ of length $k$, $1 \leq k \leq L$, be already constructed. If the last codeword $\boldsymbol{x}\left(j_{k}\right)$ is $L$-rare in $X$, then the process ends with a failure. If $k=L$ and $\boldsymbol{x}\left(j_{L}\right)$ is not $L$-rare in $X$, then the process successfully ends. Otherwise, for $k \leq L-1$, we consider $L$ indexes from $1+k(s-1)$ to $L+k(s-1)$. Since the codeword $\boldsymbol{x}\left(j_{k}\right)$ is not $L$-rare in $X$, we can find at least $L$ other codewords with the same projection on the coordinates from $1+k(s-1)$ to $L+k(s-1)$. Among them there are at most $k-1$ codewords that could be already included in the sequence $\mathcal{L}\left(j_{1}\right)$ at the previous $k-1$ steps. Therefore, there exists a codeword which has not been used. Among all such unused codewords we uniquely choose the codeword $\boldsymbol{x}\left(j_{k+1}\right)$ with the cyclically smallest index $j_{k+1}$ so that $j_{k+1}>j_{k}$ as the $(k+1)$ th element of $\mathcal{L}\left(j_{1}\right)$.

Example 1. Let $t=4$ and indexes $j_{1}=2$ and $j_{2}=5$ are already used in constructing the sequence, i.e., the first two element of the sequence $\mathcal{L}\left(j_{1}\right)$ are $(\boldsymbol{x}(2), \boldsymbol{x}(5))$. Recall that the indexes $1,5,9, \ldots$ correspond to the codeword index 1 as they have the same residue modulo $t=4$. Let codewords with indexes $3(7,11, \ldots)$ and $4(8,12, \ldots)$ be candidates to be the codeword at the third step. Then 7 , corresponding to 3 , is the cyclically smallest index so that $7>5$, and at the third stage we build the sequence $(\boldsymbol{x}(2), \boldsymbol{x}(5), \boldsymbol{x}(7))$.

Let us prove that there exists a codeword $\boldsymbol{x}\left(j_{1}\right)$ for which the described process successfully ends, i.e., as a result, we obtain a sequence $\mathcal{L}_{s}:=\mathcal{L}\left(j_{1}\right)$ without $L$-rare codewords. The process ends with a failure if and only if the codeword $\boldsymbol{x}\left(j_{k+1}\right)$ is $L$-rare at some step $k \in[L-1]$. Fix an arbitrary $L$-rare codeword $\boldsymbol{x}(j)$. Given $k \in L$, let $j_{1}$ be some element of $[t]$ so that we add $\boldsymbol{x}\left(j_{k}\right)=\boldsymbol{x}(j)$ in the sequence $\mathcal{L}\left(j_{1}\right)$ at the $k$ th step. By construction of the sequence $\mathcal{L}\left(j_{1}\right)$ we know that the codeword $\boldsymbol{x}\left(j_{k}\right)$ coincides with the codeword $\boldsymbol{x}\left(j_{k-1}\right)$ on the $L$ coordinates:

$$
1+(k-1)(s-1), 2+(k-1)(s-1), \ldots,(L-1)+(k-1)(s-1), L+(k-1)(s-1),
$$

and has the cyclically smallest index $j_{k}>j_{k-1}$ among all codeword indexes, except possibly representative indexes from $\left\{j_{1}, \ldots, j_{k-2}\right\}$. Hence, the codeword $\boldsymbol{x}\left(j_{k-1}\right)$ is the first codeword before $\boldsymbol{x}\left(j_{k}\right)$, except $\boldsymbol{x}\left(j_{1}\right), \ldots \boldsymbol{x}\left(j_{k-2}\right)$, which has the same symbols as $\boldsymbol{x}\left(j_{k}\right)$ on the $L$ coordinates (34). The number of codewords among $\boldsymbol{x}\left(j_{1}\right), \ldots, \boldsymbol{x}\left(j_{k-2}\right)$, which have the same symbols as $\boldsymbol{x}\left(j_{k}\right)$ and $\boldsymbol{x}\left(j_{k-1}\right)$ on the $L$ coordinates 34 is from 0 to $k-2$. Therefore, for fixed codeword $\boldsymbol{x}(j)$ and position $k \in[L]$, there exist at most $k-1$ possible options for $\boldsymbol{x}\left(j_{k-1}\right)$. Thus, any $L$-rare codeword $\boldsymbol{x}(j)$, uniquely chosen as the codeword $\boldsymbol{x}\left(j_{k}\right)$ in the sequence $\mathcal{L}_{s}\left(j_{1}\right)$, spoils at most $(k-1)$ ! of starting codewords $\boldsymbol{x}\left(j_{1}\right)$. In virtue of condition (32) and upper bound 31 from Lemma 1 , the code size $t>r_{L}(X) \cdot \sum_{k=0}^{L-1} k$ !. Therefore, there exists a starting codeword $\boldsymbol{x}\left(j_{1}\right)$, such that the sequence $\mathcal{L}\left(j_{1}\right)$ will be successfully constructed.

Lemma 3. For any list-decoding $(s, L, q)$-code $X$ of length $N=s+L-1$, the size $t$ of the code $X$ is upper bounded as follows:

$$
t \leq(s+L-1) L q^{L} \sum_{k=0}^{L-1} k ! .
$$


Proof of Lemma 3 Consider an arbitrary list-decoding $(s, L, q)$-code $X$ of the length $N=s+L-1$. We prove the claim of this lemma by contradiction. Assume that $t>(s+L-1) L q^{L} \sum_{k=0}^{L-1} k$ !. In virtue of Lemma 2 , we can construct the sequence $\mathcal{L}_{s}=\left(\boldsymbol{x}\left(j_{1}\right), \ldots, \boldsymbol{x}\left(j_{L}\right)\right)$ so that there is no $L$-rare codeword in $\mathcal{L}_{s}$, and the property (33) holds. Let $J=\left\{j_{1}, \ldots, j_{L}\right\}$ be the set of codeword indexes. Without loss of generality, we may assume the sequence $\left(j_{1}, j_{2}, \ldots, j_{L}\right)$ is lexicographically ordered or $j_{k}<j_{k+1}$ for $k \in[L-1]$, since, otherwise, we can take $[30] j_{k+1}$ as $j_{k+1}+t\left\lceil j_{k} / t\right\rceil$.

Now we shall find an $s$-collection $I=\left\{i_{1}, \ldots, i_{s}\right\} \subset[t] \backslash J$ consisting of codeword indexes such that $U\left(\boldsymbol{x}\left(i_{1}\right), \ldots, \boldsymbol{x}\left(i_{s}\right)\right)$ covers $L$ codewords $\{x(j), j \in J\}$. Recall that by covering we mean that, for any pair $(j, n), j \in J, n \in[N]$, there exists $i \in I$ so that the symbol $x_{n}(j)=x_{n}(i)$. Define a lexicographically ordered sequence $\mathcal{P}$ of pairs so that the first $s+L-1$ pairs are from $\left(j_{1}, 1\right)$ to $\left(j_{1}, s+L-1\right)$, and the following $(s-1)(L-1)$ pairs are of the form $\left(j_{k}, n\right)$, where $n$ runs over all row indexes from $L+1+(k-1)(s-1)$ to $L+k(s-1)$, i.e.,

$$
\begin{aligned}
\mathcal{P} \triangleq\left(\left(j_{1}, 1\right),\left(j_{1}, 2\right), \ldots,\right. & \left(j_{1}, L+s-1\right), \\
& \left(j_{2}, L+1+(s-1)\right),\left(j_{2}, L+2+(s-1)\right), \ldots,\left(j_{2}, L+2(s-1)\right), \ldots, \\
& \left.\left(j_{L}, L+1+(L-1)(s-1)\right),\left(j_{L}, L+2+(L-1)(s-1)\right) \ldots,\left(j_{L}, s L\right)\right) .
\end{aligned}
$$

From (33) it follows that if, for any pair $(j, n)$ in $\mathcal{P}$, there exists $i \in I$ so that the symbol $x_{n}(j)=x_{n}(i)$, then the $s$-collection $I$ is a required one. It remains to find appropriate $I$. Notice that the length of $\mathcal{P}$ is $s L$, and the second number in pairs goes from 1 to $s L$. Divide the sequence $\mathcal{P}$ into $s$ subsequences of length $L$ so that $\mathcal{P}=\left(\mathcal{P}_{1}, \ldots, \mathcal{P}_{s}\right)$. Let

$$
\mathcal{P}_{k} \triangleq\left(\left(j_{k_{1}},(k-1) L+1\right),\left(j_{k_{2}},(k-1) L+2\right), \ldots,\left(j_{k_{L}}, k L\right)\right) .
$$

It is easy to check that the projection $\boldsymbol{x}\left(j_{k_{L}}\right)$ (the codeword index is the same as the first number in the last pair of $\mathcal{P}_{k}$ ) on the coordinates $(k-1) L+1,(k-1) L+2, \ldots, k L$ is

$$
\boldsymbol{x}_{(k-1) L+1}^{k L}\left(j_{k_{L}}\right)=\left(x_{(k-1) L+1}\left(j_{k_{1}}\right), x_{(k-1) L+2}\left(j_{k_{2}}\right), \ldots, x_{k L}\left(j_{k_{L}}\right)\right) \text {. }
$$

From Lemma 2, it follows that the codeword $\boldsymbol{x}\left(j_{k_{L}}\right)$ is not $L$-rare. Therefore, we can find an index $i_{k}, i_{k} \notin J$, and the corresponding codeword $\boldsymbol{x}\left(i_{k}\right)$ such that the projections of $\boldsymbol{x}\left(i_{k}\right)$ and $\boldsymbol{x}\left(j_{k_{L}}\right)$ on the coordinates $(k-1) L+1,(k-1) L+2, \ldots, k L$ are the same, i.e.,

$$
\boldsymbol{x}_{(k-1) L+1}^{k L}\left(i_{k}\right)=\boldsymbol{x}_{(k-1) L+1}^{k L}\left(j_{k_{L}}\right) .
$$

Since there are $s$ subsequences $\mathcal{P}_{k}$, which form $\mathcal{P}$, we can find at most $s$ different $i_{k}$ so that $U\left(\boldsymbol{x}\left(i_{1}\right), \ldots, \boldsymbol{x}\left(i_{s}\right)\right)$ covers $L$ codewords $\{\boldsymbol{x}(j), j \in J\}$. This contradiction finishes the proof of Lemma 3

Lemmas 2 and 3 are intuitively illustrated by the following example.

Example 2. Let $L=4, s=2$ and $N=L+s-1=5$. Then four $q$-ary codewords $\boldsymbol{x}\left(j_{k}\right), \boldsymbol{x}\left(j_{k}\right) \in \mathcal{A}_{q}^{5}, k \in\{1,2,3,4\}$, satisfying the equalities (33) can be written in the form:

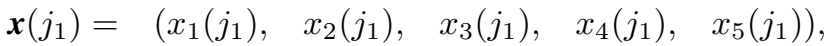

$$
\begin{aligned}
& \boldsymbol{x}\left(j_{2}\right)=\left(y_{2}, \quad x_{2}\left(j_{1}\right), \quad x_{3}\left(j_{1}\right), \quad x_{4}\left(j_{1}\right), \quad x_{5}\left(j_{1}\right)\right),
\end{aligned}
$$

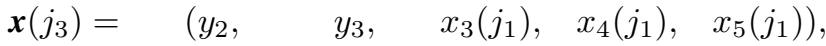

$$
\begin{aligned}
& \boldsymbol{x}\left(j_{4}\right)=\left(y_{2}, \quad y_{3}, \quad y_{4}, \quad x_{4}\left(j_{1}\right), \quad x_{5}\left(j_{1}\right)\right) .
\end{aligned}
$$

These codewords are covered by $U\left(\boldsymbol{x}\left(i_{1}\right), \boldsymbol{x}\left(i_{2}\right)\right)$, where two $q$-ary codewords $\boldsymbol{x}\left(i_{1}\right), \boldsymbol{x}\left(i_{2}\right) \in \mathcal{A}_{q}^{5}$ are based on the property 36 and can be written in the form:

$$
\begin{aligned}
& \boldsymbol{x}\left(i_{1}\right)=\left(x_{1}\left(j_{1}\right), \quad x_{2}\left(j_{1}\right), \quad x_{3}\left(j_{1}\right), \quad x_{4}\left(j_{1}\right), \quad a_{1}\right), \\
& \boldsymbol{x}\left(i_{2}\right)=\left(\begin{array}{lllll}
\left(y_{2},\right. & y_{3}, & y_{4}, & a_{2} & \left.x_{5}\left(j_{1}\right)\right) .
\end{array}\right.
\end{aligned}
$$

To complete the proof of Theorem 5 . consider an arbitrary list-decoding $(s, L, q)$-code $X$ of length $N, N>s+L-1$, and size $t$. Divide each codeword of the code $X$ into $s+L-1$ parts of sizes $n_{i}$, where $\left[\frac{N}{s+L-1}\right\rfloor \leq n_{i} \leq\left\lceil\frac{N}{s+L-1}\right\rceil, i \in[s+L-1]$. The number of different parts is upper bounded by $q^{\left\lfloor\frac{N}{s+L-1}\right\rfloor}+q^{\left\lceil\frac{N}{s+L-1}\right\rceil}$. Replace each part of each codeword with a unique symbol from the $Q$-ary alphabet of the size $Q \triangleq 2 q^{\left.\frac{N}{s+L-1}\right\rceil}$. It is easy to see that the code $X^{\prime}$, obtained after replacements, is a $Q$-ary list-decoding $(s, L, Q)$-code of length $N=s+L-1$ and size $t$. Thus, the inequality (35) of Lemma 3 implies that the size

$$
t \leq(s+L-1) L \sum_{n=0}^{L-1} n ! 2^{L} q^{L\left\lceil\frac{N}{s+L-1}\right\rceil .}
$$

This upper bound immediately leads to 29 .

\section{APPENDIX}

In this section, we first introduce a probabilistic relaxation of separable codes called almost separable codes, and then give random coding bounds on the error exponent of almost separable codes and on the rate of separable codes for any $f$-MAC. 


\section{A. Notations and Definitions}

Given the symmetric $f$-MAC and a $q$-ary code $X$, a message e $\in\left(\begin{array}{c}{[t]} \\ s\end{array}\right)$ is said to be bad for the code $X$, if there exists a message $\mathbf{e}^{\prime} \neq \mathbf{e}$ such that $z^{(f)}\left(\mathbf{e}^{\prime}, X\right)=z^{(f)}(\mathbf{e}, X)$. If the unknown message $\mathbf{e}$ is interpreted as the random vector taking equiprobable values in the set $\left(\begin{array}{c}{[t]} \\ s\end{array}\right)$, then the relative number of "bad" messages among all $\left(\begin{array}{c}t \\ s\end{array}\right)=\left|\left(\begin{array}{c}{[t]} \\ s\end{array}\right)\right|$ messages can be considered as the error probability $\epsilon^{(f)}(X, s)$ of code $X$ for the brute force decoding.

Definition 4. A code $X$ is called an almost $s$-separable code for the $f$-MAC with the error probability $\epsilon$ if the relative number of bad messages in the code $X$ is at most $\epsilon$, that is $\epsilon^{(f)}(X, s) \leq \epsilon$.

Let us introduce the classical notation of the error exponent and the capacity.

Definition 5. Fix a parameter $R>0$. Define the error probability for almost $s$-separable codes

$$
\epsilon^{(f)}(s, q, R, N) \triangleq \min _{X: t=\left\lfloor 2^{R N}\right\rfloor} \epsilon^{(f)}(X, s),
$$

where the minimum is taken over all $q$-ary codes of length $N$ and size $t$. The function

$$
E^{(f)}(s, q, R) \triangleq \varlimsup_{N \rightarrow \infty} \frac{-\log _{2} \epsilon^{(f)}(s, q, R, N)}{N}
$$

will be referred to as the error exponent for almost $s$-separable codes. The quantity

$$
C^{(f)}(s, q)=\sup \left\{R: E^{(f)}(s, q, R)>0\right\}
$$

is called the capacity of almost $s$-separable codes.

We again emphasize that the rate of separable codes is upper bounded by the capacity of almost separable codes.

Proposition 1. [27]. The rate $R^{(f)}(s, q)$ of s-separable codes for the symmetric $f$-MAC satisfies the inequality

$$
R^{(f)}(s, q) \leq C^{(f)}(s, q) \triangleq \frac{\max _{\boldsymbol{p}} H_{\boldsymbol{p}}^{(f)}(s, q)}{s},
$$

where $H_{p}^{(f)}(s, q)$ is the Shannon entropy (17) of the output of the f-MAC for the given input probability distribution $\boldsymbol{p}$.

\section{B. Random Coding Error Exponent for the f-MAC}

Let the symbol $\mathcal{P}_{N}^{(f)}\left(s, t,\left(N_{0}, \ldots, N_{q-1}\right)\right)$ denote the average error probability over the fixed composition ensemble (briefly, $F C$-ensemble) of $t$ independent $q$-ary codewords $\boldsymbol{x}(i)$ with the same type $T(\boldsymbol{x}(i))=\left(N_{0}, \ldots, N_{q-1}\right)$. By a similar symbol $\mathcal{P}_{N}^{(f)}(s, t, \boldsymbol{p})$ we will denote the average error probability over the completely randomized ensemble (briefly, $C R$-ensemble) of $q$-ary codes $X=\left(x_{i}(j)\right)$ with independent components $x_{i}(j)$ having the same distribution $\boldsymbol{p}$, i.e., $\operatorname{Pr}\left\{x_{i}(j)=x\right\} \triangleq p(x)$, $i \in[N], j \in[t], x \in \mathcal{A}_{q}$.

Let a symmetric $f$-MAC is represented as the conditional probability $\tau^{(f)}\left(z \mid x_{1}^{s}\right)$, that is

$$
\tau^{(f)}\left(z \mid x_{1}^{s}\right)= \begin{cases}1, & z=f\left(x_{1}^{s}\right) \\ 0, & z \neq f\left(x_{1}^{s}\right)\end{cases}
$$

To formulate the results about the logarithmic asymptotic behavior of probabilities $\mathcal{P}_{N}^{(f)}\left(s, t,\left(N_{0}, ., N_{q-1}\right)\right)$ and $\mathcal{P}_{N}^{(f)}(s, t, \boldsymbol{p})$, we need the following auxiliary notations [26]. Let

$$
\tau \triangleq\left\{\tau\left(x_{1}^{s}, z\right): \tau\left(x_{1}^{s}, z\right) \geq 0, \sum_{x_{1}^{s}, z} \tau\left(x_{1}^{s}, z\right)=1\right\}
$$

be a probability distribution on the Cartesian product $\mathcal{A}_{q}^{s} \times Z$. Using the standard symbols for the conditional probabilities of the distribution $\tau$, we abbreviate by

$$
\{\tau\}^{(f)} \triangleq\left\{\tau: \tau^{(f)}\left(z \mid x_{1}^{s}\right)=0 \Rightarrow \tau\left(z \mid x_{1}^{s}\right)=0\right\}
$$

the subset of probability distributions $\tau$ 37 such that the conditional probability $\tau\left(z \mid x_{1}^{s}\right)=0$ is implied by $\tau^{(f)}\left(z \mid x_{1}^{s}\right)=0$.

Introduce the $\cup$-convex information-theoretic functions of the argument $\tau \in\{\tau\}^{(f)}$ :

$$
\mathcal{H}^{(f)}(\boldsymbol{p}, \tau) \triangleq \sum_{x_{1}^{s} \cdot z} \tau\left(x_{1}^{s}, z\right) \ln \frac{\tau\left(x_{1}^{s}, z\right)}{\tau^{(f)}\left(z \mid x_{1}^{s}\right) \cdot \prod_{k=1}^{s} p\left(x_{k}\right)}, \quad I_{m}(\boldsymbol{p}, \tau) \triangleq \sum_{x_{1}^{s} \cdot z} \tau\left(x_{1}^{s}, z\right) \ln \frac{\tau\left(x_{1}^{m} \mid x_{m+1}^{s}, z\right)}{\prod_{k=1}^{m} p\left(x_{k}\right)}, m \in[s] .
$$

From [17), it follows that the distribution

$$
\tau_{p}^{(f)} \triangleq\left\{\tau^{(f)}\left(z \mid x_{1}^{s}\right) \cdot \prod_{k=1}^{s} p\left(x_{k}\right), x_{1}^{s} \in \mathcal{A}_{q}^{s}, z \in Z\right\} \in\{\tau\}^{(f)}
$$


and the functions 39 , satisfy the equalities

$$
\mathcal{H}^{(f)}\left(\boldsymbol{p}, \tau_{\boldsymbol{p}}^{(f)}\right)=0, \quad I_{s}\left(\boldsymbol{p}, \tau_{\boldsymbol{p}}^{(f)}\right)=H_{\boldsymbol{p}}^{(f)}(s, q) .
$$

Now we are ready to state two random coding bounds on the error exponent $E^{(f)}(s, q, R)$.

Proposition 4. [26], [27]. Let $s \geq 2, q \geq 2, R>0$ be fixed and the entropy $H_{p}^{(f)}(s, q)$ of a fixed distribution $\boldsymbol{p}$ is defined by (17). If code parameters $N, t \rightarrow \infty$ such that

$$
\frac{\ln t}{N} \sim R, \quad \frac{N_{x}}{N} \sim p(x), x \in \mathcal{A}_{q}, \quad s, q-\text { const },
$$

then for the FC-ensemble there exists

$$
\lim _{N \rightarrow \infty} \frac{-\ln \mathcal{P}_{N}^{(f)}\left(s, t,\left(N_{0}, ., N_{q-1}\right)\right)}{N} \triangleq E_{F C}^{(f)}(s, q, R, \boldsymbol{p})>0, \quad 0<R<\frac{H_{p}^{(f)}(s, q)}{s},
$$

and for the CR-ensemble there exists

$$
\lim _{N \rightarrow \infty} \frac{-\ln \mathcal{P}_{N}^{(f)}(s, t, \boldsymbol{p})}{N} \triangleq E_{C R}^{(f)}(s, q, R, \boldsymbol{p})>0, \quad 0<R<\frac{H_{p}^{(f)}(s, q)}{s} .
$$

For any fixed $\boldsymbol{p}$, the positive monotonically decreasing functions $E_{F C}^{(f)}(s, q, R, \boldsymbol{p})$ and $E_{C R}^{(f)}(s, q, R, \boldsymbol{p})$ are $\cup$-convex functions of the parameter $R>0$ of the following form:

$$
\begin{gathered}
E_{F C}^{(f)}(s, q, R, \boldsymbol{p}) \triangleq \min _{m \in[s]} E_{F C}^{(f)}(s, q, R, \boldsymbol{p}, m), \\
E_{F C}^{(f)}(s, q, R, \boldsymbol{p}, m) \triangleq \min _{\{\tau\}^{(f)}(\boldsymbol{p})}\left\{\mathcal{H}^{(f)}(\boldsymbol{p}, \tau)+\left[I_{m}(\boldsymbol{p}, \tau)-m R\right]^{+}\right\},
\end{gathered}
$$

and

$$
\begin{gathered}
E_{C R}^{(f)}(s, q, R, \boldsymbol{p}) \triangleq \min _{m \in[s]} E_{C R}^{(f)}(s, q, R, \boldsymbol{p}, m), \\
E_{C R}^{(f)}(s, q, R, \boldsymbol{p}, m) \triangleq \min _{\{\tau\}(f)}\left\{\mathcal{H}^{(f)}(\boldsymbol{p}, \tau)+\left[I_{m}(\boldsymbol{p}, \tau)-m R\right]^{+}\right\} .
\end{gathered}
$$

The minimum in 42 is taken over the subset $\{\tau\}^{(f)}(\boldsymbol{p})$ of distributions $\{\tau\}^{(f)}$ (38) for which the marginal probabilities on $x_{k}$ are fixed and coincide with $p\left(x_{k}\right), k \in[s]$, i.e.,

$$
\{\tau\}^{(f)}(\boldsymbol{p}) \triangleq\left\{\tau: \tau \in\{\tau\}^{(f)} \quad \text { and } \quad \sum_{x_{1}^{k-1}} \sum_{x_{k+1}^{s}} \sum_{z} \tau\left(x_{1}^{s}, z\right)=p\left(x_{k}\right), k \in[s]\right\} .
$$

The minimum in (43) is taken over the set of all distributions (38). In addition, for any p, the error exponent of almost separable codes for the $f-M A C$

$$
E^{(f)}(s, q, R) \geq E_{F C}^{(f)}(s, q, R, \boldsymbol{p}), \quad E^{(f)}(s, q, R) \geq E_{C R}^{(f)}(s, q, R, \boldsymbol{p}) .
$$

Remark 3. Propositions 14 and the properties of the random error exponents $(40)$ and 41 , were formulated and proved in the papers [27] and [26] for the particular binary case $q=2$ only. In the general case $q \geq 2$, we omit the proofs because one can check that the given results are based on the same methods developed in [27] and [26]. Here we only note that for the symmetric $f$-MAC, definitions (42)-(44) leads to the inequality

$$
E_{C R}^{(f)}(s, q, R, \boldsymbol{p}) \leq E_{F C}^{(f)}(s, q, R, \boldsymbol{p}) .
$$

Introduce the function

$$
E_{F C}^{(f)}(s, q, R) \triangleq \max _{\boldsymbol{p}} E_{F C}^{(f)}(s, q, R, \boldsymbol{p})>0
$$

if $0<R<C^{(f)}(s, q)$, where $C^{(f)}(s, q)$ is defined in the right-hand side 18 . Hence, Propositions 1 and 4 imply that the number $C^{(f)}(s, q)$ can be considered as the Shannon capacity of separable $(s, q)$-codes for the symmetric $f$-MAC [39].

The following statement called the random coding lower bound on the rate $R^{(f)}(s, q)$ of $s$-separable $q$-ary codes for the symmetric $f$-MAC can be obtained as a consequence of Proposition 4.

Proposition 5. [26]. The rate $R^{(f)}(s, q)$ of s-separable q-ary codes for the symmetric $f$-MAC satisfies the inequality

$$
R^{(f)}(s, q) \geq \underline{R}^{(f)}(s, q), \quad s \geq 2, q \geq 2,
$$


where for any fixed distribution $\boldsymbol{p}$ the lower bound $\underline{R}^{(f)}(s, q)$ can be represented in the form

$$
\underline{R}^{(f)}(s, q) \triangleq \min _{m \in[s]} \frac{E_{F C}^{(f)}(s, q, 0, \boldsymbol{p}, m)}{s+m-1}=\min _{m \in[s]} \frac{\min _{\{\tau\}^{(f)}(\boldsymbol{p})}\left\{\mathcal{H}^{(f)}(\boldsymbol{p}, \tau)+I_{m}(\boldsymbol{p}, \tau)\right\}}{s+m-1}
$$

or in the form

$$
\underline{R}^{(f)}(s, q) \triangleq \min _{m \in[s]} \frac{E_{C R}^{(f)}(s, q, 0, \boldsymbol{p}, m)}{s+m-1}=\min _{m \in[s]} \frac{\min _{\{\tau\}^{(f)}}\left\{\mathcal{H}^{(f)}(\boldsymbol{p}, \tau)+I_{m}(\boldsymbol{p}, \tau)\right\}}{s+m-1}
$$

In paper [26], Proposition 5] was proved for the particular case of the B-MAC with binary $(q=2)$ alphabet only. For the arbitrary symmetric $f$-MAC, one can use the same arguments. The asymptotic lower bound on the rate $R^{(d i s j)}(s)$ for the disjunctive MAC formulated in Sect. III-B was actually obtained in [26] as a nontrivial consequence of Proposition 5.

\section{REFERENCES}

[1] S. C. Chang and J. K. Wolf, "On the $T$-user $M$-frequency noiseless multiple-access channel with and without intensity information," IEEE Trans. Inform. Theory, vol. 27, no. 1, pp. 41-48, 1981 .

[2] I. Csiszar and J. Körner, Information theory: coding theorems for discrete memoryless systems. Cambridge University Press, 2011.

[3] M. Cheng and Y. Miao, "On anti-collusion codes and detection algorithms for multimedia fingerprinting," IEEE transactions on information theory, vol. 57, no. 7, pp. 4843-4851, 2011.

[4] E. Egorova and V. Potapova, "Signature codes for a special class of multiple access channel," in Problems of Redundancy in Information and Control Systems (REDUNDANCY), 2016 XV International Symposium, pp. 38-42, IEEE, 2016.

[5] D. Boneh and J. Shaw, "Collusion-secure fingerprinting for digital data," IEEE Transactions on Information Theory, vol. 44, no. 5, pp. 1897-1905, 1998.

[6] M. L. Fredman and J. Komlós, "On the size of separating systems and families of perfect hash functions," SIAM J. Algebraic Discrete Methods, vol. 5, no. 1, pp. 61-68, 1984 .

[7] K. Mehlhorn, "Sorting and searching, volume 1 of data structures and algorithms," 1984.

[8] M. Cheng, L. Ji, and Y. Miao, "Separable codes," IEEE Transactions on Information Theory, vol. 58, no. 3, pp. 1791-1803, 2012.

[9] F. Gao and G. Ge, "New bounds on separable codes for multimedia fingerprinting," IEEE Trans. Inform. Theory, vol. 60, no. 9, pp. 5257-5262, 2014.

[10] S. R. Blackburn, "Probabilistic existence results for separable codes," IEEE Trans. Inform. Theory, vol. 61, no. 11, pp. 5822-5827, 2015.

[11] C. Shangguan, X. Wang, G. Ge, and Y. Miao, "New bounds for frameproof codes," IEEE Trans. Inform. Theory, vol. 63, no. 11, pp. 7247-7252, 2017.

[12] J. N. Staddon, D. R. Stinson, and R. Wei, "Combinatorial properties of frameproof and traceability codes," IEEE transactions on information theory, vol. 47, no. 3, pp. 1042-1049, 2001.

[13] S. R. Blackburn, "Frameproof codes," SIAM Journal on Discrete Mathematics, vol. 16, no. 3, pp. 499-510, 2003.

[14] A. G. Dyachkov, I. V. Vorobyev, N. Polyanskii, and V. Y. Shchukin, "Cover-free codes and separating system codes," Designs, Codes and Cryptography, vol. 82, no. 1-2, pp. 197-209, 2017.

[15] D. R. Stinson, R. Wei, and K. Chen, "On generalized separating hash families," J. Combin. Theory Ser. A, vol. 115, no. 1, pp. 105-120, 2008.

[16] L. Bassalygo, M. Burmester, A. Dyachkov, and G. Kabatianskii, "Hash codes," in Proc. IEEE Int'l Symp. Inf. Theory (ISIT), pp. 174-174, 1997.

[17] W. Kautz and R. Singleton, "Nonrandom binary superimposed codes," IEEE Trans. Inform. Theory, vol. 10, no. 4, pp. 363-377, 1964.

[18] A. D'yachkov and V. Rykov, "A survey of superimposed code theory," Problems Control Inform. Theory/Problemy Upravlen. Teor. Inform., vol. 12, no. 4, pp. 229-242, 1983.

[19] A. Dyachkov, "An upper bound for hash codes," in Conference "Computer Science and Information Technologies", pp. 219-221, 1997.

[20] J. Körner and K. Marton, "New bounds for perfect hashing via information theory," European J. Combin., vol. 9, no. 6, pp. 523-530, 1988.

[21] L. A. Bassalygo and M. S. Pinsker, "Evaluation of the asymptotics of the summarized capacity of an $m$-frequency $t$-user noiseless multiple-access channel," Probl. Inf. Trans., vol. 36, no. 2, pp. 91-97, 2000.

[22] L. Wilhelmsson and K. Zigangirov, "On the asymptotic capacity of a multiple-access channel," Probl. Inf. Trans., vol. 33, no. 1, pp. 9-16, 1997.

[23] A. H. Vinck and K. J. Keuning, "On the capacity of the asynchronous $t$-user $m$-frequency noiseless multiple-access channel without intensity information," IEEE Trans. Inform. Theory, vol. 42, no. 6, pp. 2235-2238, 1996.

[24] Y. Erlich, A. Gordon, M. Brand, G. J. Hannon, and P. P. Mitra, "Compressed genotyping," IEEE Trans. Inform. Theory, vol. 56, no. 2, pp. 706-723, 2010.

[25] D.-Z. Du and F. K. Hwang, Combinatorial group testing and its applications, vol. 12 of Series on Applied Mathematics. World Scientific Publishing Co., Inc., River Edge, NJ, second ed., 2000.

[26] A. D'yachkov, Lectures on Designing Screening Experiments, vol. 10 of Lect. Note Ser. Pohang, Korea: Pohang Univ. of Science and Technology (POSTECH), 2003.

[27] A. G. D'yachkov and A. Rashad, "Universal decoding for random design of screening experiments," Microelectronics and Reliability, vol. 29, no. 6, pp. 965-971, 1989

[28] M. Malyutov, "The separating property of random matrices," Mathematical Notes, vol. 23, no. 1, pp. 84-91, 1978.

[29] D. Coppersmith and J. B. Shearer, "New bounds for union-free families of sets," Electron. J. Combin., vol. 5, pp. Research Paper 39, $16,1998$.

[30] A. G. D'yachkov, I. V. Vorob'ev, N. A. Polyansky, and V. Y. Shchukin, "Bounds on the rate of disjunctive codes," Probl. Inf. Transm., vol. 50, no. 1, pp. 27-56, 2014. Translation of Problemy Peredachi Informatsii 50 (2014), no. 1, 31-63.

[31] A. M. Rashad, "On symmetrical superimposed codes," J. Inform. Process. Cybernet., vol. 25, no. 7, pp. 337-341, 1989.

[32] V. Y. Shchukin, "List decoding for a multiple access hyperchannel," Probl. Inf. Trans., vol. 52, no. 4, pp. 329-343, 2016.

[33] A. D'yachkov, V. Rykov, C. Deppe, and V. Lebedev, "Superimposed codes and threshold group testing," in Information theory, combinatorics, and search theory, vol. 7777 of Lecture Notes in Comput. Sci., pp. 509-533, Springer, Heidelberg, 2013.

[34] A. De Bonis and U. Vaccaro, "Optimal algorithms for two group testing problems, and new bounds on generalized superimposed codes," IEEE Trans. Inform. Theory, vol. 52, no. 10, pp. 4673-4680, 2006.

[35] A. Dyachkov, "On a search model of false coins," in Topics in Information Theory (ed. Csiszar, I.-Elias, P.). Colloqua Mathematica Sociatotis Janos Bolyai.

[36] A. G. D’yachkov and V. V. Rykov, "On a coding model for a multiple-access adder channel," Problemy Peredachi Informatsii, vol. 17, no. 2, pp. 26-38, 1981.

[37] P. Mateev, "On the entropy of the multinomial distribution," Theory of Probability \& Its Applications, vol. 23, no. 1, pp. 188-190, 1978.

[38] A. Naor and J. Verstraëte, "A note on bipartite graphs without 2k-cycles," Combin. Probab. Comput., vol. 14, no. 5-6, pp. 845-849, 2005.

[39] M. B. Malyutov and P. S. Mateev, "Planning of screening experiments for a nonsymmetric response function," Mathematical notes of the Academy of Sciences of the USSR, vol. 27, no. 1, pp. 57-68, 1980. 